DAMTP-HEP-94/85

\title{
The Space of Local Operators in Perturbed Conformal Field Theories
}

\author{
A. Koubek用 \\ Department of Applied Mathematics and Theoretical Physics, \\ Silver Street, \\ CB3 9EW Cambridge, \\ $U K$
}

\begin{abstract}
The space of local operators in massive deformations of conformal field theories is analysed. For several model systems it is shown that one can define chiral sectors in the theory, such that the chiral field content is in a one-to-one correspondence with that of the underlying conformal field theory. The full space of operators consists of the descendent spaces of all scalar fields. If the theory contains asymptotic states which satisfy generalised statistics, the form factor equations admit in addition also solutions corresponding to the descendent spaces of the para-fermionic operators of the same spin as the asymptotic states. The derivation of these results uses $q$-sum expressions for the characters and $q$-difference equations used in proving Rogers-Ramanujan type identities.
\end{abstract}

October 1994

*E-mail: a.koubek@amtp.cam.ac.uk 


\section{Introduction}

During the last several years the theory of completely integrable models in $(1+1)$ dimensions has experienced a rapid development. Especially the study of conformal field theories (CFT), which resulted in the solution of several classes of such theories, has allowed a deep understanding of the critical points describing second order phase transitions. Unfortunately, less is known for off-critical integrable field theories. Their solution should give a description of the scaling region around critical points and allow a deeper understanding of standard techniques used in quantum field theory, such as the renormalisation group or perturbation theory.

In this article we will study so-called perturbed conformal field theories, i.e. models which can be viewed as perturbations of a conformal field theory by a relevant operator. The perturbation is chosen in a way that the theory remains integrable also away from the critical point. This description proves useful, since many properties of the conformal theory can be preserved or 'deformed' off-criticality. As, for example, the quantum group structure, which becomes an affine one in the perturbation process, or the conserved charges.

The ultimate goal in the investigation of these systems is to solve them, that is to determine the algebra of local operators and their correlation functions. In general conformal field theories perturbed by the operators $\phi_{1,2}, \phi_{2,1}$ and $\phi_{1,3}$

$$
H=H_{C F T}+\lambda \int d^{2} x \phi(x)
$$

are driven into a massive regime. It has been shown by A. Zamolodchikov [1], that such theories can be described by the exact scattering matrix. A lot of progress has been made in determining in general the on-shell structure and understanding its algebraic origin.

The understanding of the off-shell structure of these massive integrable theories is less complete. Knowing the exact $S$-matrix of a theory one can calculate the matrix-elements of local operators, called form factors. They are determined from the knowledge of the on-shell structure of the theory, through the form factor equations. This is a remarkable property, since the on-shell structure of the theory does not contain any information about local fields. In fact it is described in terms of asymptotic states. 
The form factor equations do not refer to a specific operator. This on the one hand makes the identification of operators a difficult task. Apart from the fundamental fields of a theory (as the energy-momentum tensor or conserved currents) which have additional properties, it is difficult to identify a specific operator from the knowledge of its form factors. This is true especially in this case, where we are interested in the comparison with the conformal field theory in the ultraviolet limit. In order to achieve such an identification, one would need to calculate correlation functions, which involves the summing up of the form factor series, a problem which has not been solved for interacting models.

The above mentioned arbitrariness in the form factor equations allows the determination of the space of local operators of the theory. Since the equations must be valid for all local fields of the theory, one can specify the field content by determining the independent solutions of the form factor equations. In this article we will use precisely this fact, and find the space of local operators for some simple massive theories. They have the distinguishing property that they contain only scalar asymptotic states, and can be described as perturbed minimal conformal field theories.

Beforehand, it is not clear what kind of structure one should expect to find in the perturbed conformal theories. Since the perturbation theory defined by (1.1) is superrenormalizable, only a finite number of counter terms is involved in the renormalisation procedure, which should leave unchanged the structure of the operator algebra of the theory [1]. On the other hand the most salient feature of the critical theory is its Virasoro algebra symmetry, which is broken in the massive theory and one expects drastic changes in the off-critical theory. In the critical theory left and right chiral sectors (corresponding to the components $T$ and $\bar{T}$ of the energy momentum tensor) are independent which certainly is not true in the deformed theory. Also the structure of the conserved charges changes in the course of the perturbation.

The result of this study will be that the structure of the massive theory resembles much the one of the conformal theory. We will define a grading in the space of operators which allows a formal definition of chiral components. The so-defined chiral structure of the deformed theory becomes isomorphic to the conformal one. In CFT on the real line there are no constraints on which combinations of the two chiral dimensions of the operators 
can enter the partition function, apart that they should form a closed operator algebra. In the massive model we have an additional locality requirement, deriving from the form factor equations. As will be shown in the following sections, only specific combinations of chiral dimensions are present in the massive theory.

\subsection{The Form Factor Bootstrap Approach}

Let us review some general properties of the form factors [2, 3, 6 , 5]. We parametrise the momenta in terms of the masses of the particles $m_{i}$ and the rapidity variables $\beta_{i}$ as

$$
p_{i}^{0}=m_{i} \cosh \beta_{i}, p_{i}^{1}=m_{i} \sinh \beta_{i}
$$

Form factors are defined as matrix elements of local operators $\mathcal{O}$ between the vacuum and the set of asymptotic states,

$$
\mathcal{F}_{\epsilon_{1}, \epsilon_{2}, \ldots, \epsilon_{n}}^{\mathcal{O}}\left(\beta_{1}, \beta_{2}, \ldots, \beta_{n}\right)=\left\langle 0|\mathcal{O}(0,0)| Z_{\epsilon_{1}}\left(\beta_{1}\right), Z_{\epsilon_{2}}\left(\beta_{2}\right), \ldots, Z_{\epsilon_{n}}\left(\beta_{n}\right)\right\rangle_{\text {in }}
$$

Properties deriving from basic principles of quantum field theory, such as crossing and unitarity, and the properties of the Fadeev-Zamolodchikov operators $Z_{\epsilon}(\beta)$ allow the construction of these matrix elements. For the case of scalar particles these functional equations known as the form factor axioms are:

1) Watson's equations

$$
\begin{gathered}
\mathcal{F}_{\epsilon_{1} \ldots \epsilon_{i} \epsilon_{i+1} \ldots \epsilon_{n}}^{\mathcal{O}}\left(\beta_{1}, \ldots, \beta_{i}, \beta_{i+1}, \ldots \beta_{n}\right)= \\
S_{\epsilon_{i} \epsilon_{i+1}}\left(\beta_{i}-\beta_{i+1}\right) \mathcal{F}_{\epsilon_{1} \ldots \epsilon_{i+1} \epsilon_{i} \ldots \epsilon_{n}}^{\mathcal{O}}\left(\beta_{1}, \ldots, \beta_{i+1}, \beta_{i}, \ldots \beta_{n}\right), \\
\mathcal{F}_{\epsilon_{1} \epsilon_{2} \ldots \epsilon_{n}}^{\mathcal{O}}\left(\beta_{1}+2 \pi i, \beta_{2}, \ldots, \beta_{n}\right)=e^{2 i \pi\left(s_{\epsilon_{n}}+w_{\epsilon_{n}}+\sum w_{\epsilon_{i} \epsilon_{n}}\right)} \mathcal{F}_{\epsilon_{2} \ldots \epsilon_{n} \epsilon_{1}}^{\mathcal{O}}\left(\beta_{2}, \ldots, \beta_{n}, \beta_{1}\right),
\end{gathered}
$$

where $s_{\epsilon_{n}}$ denotes the spin of the particle $\epsilon_{n}$ and $w_{\epsilon}$ denotes the mutual locality index between the field $\mathcal{O}$ and the field creating the asymptotic state $Z_{\epsilon}$. Similarly $w_{\epsilon_{i} \epsilon_{n}}$ denotes the generalised statistics, which the operators creating the particles $i$ and $n$ satisfy. It is 
determined by $\lim _{\beta \rightarrow \infty} S_{n i}(\beta)$. This phase factor is necessary since for some theories we will consider, the particles will not become free particles for $\beta \rightarrow \pm \infty$, but will satisfy

$$
Z_{\epsilon_{i}} Z_{\epsilon_{j}}=e^{2 i \pi w_{i j}} Z_{\epsilon_{j}} Z_{\epsilon_{i}}
$$

Therefore whenever one continues the form factors analytically, one needs to take these commutation properties into account. For theories containing degenerate particles this statistics is governed by the $R$-matrix defining the ultraviolet limit of the scattering theory [5]. Alternatively these phases can be taken in account by assigning non-trivial charge conjugation to the operators creating the asymptotic states.

2) Relativistic invariance

$$
\mathcal{F}_{\epsilon_{1} \ldots \epsilon_{n}}^{\mathcal{O}}\left(\beta_{1}+\Lambda, \ldots, \beta_{n}+\Lambda\right)=e^{s \Lambda} \mathcal{F}_{\epsilon_{1} \ldots \epsilon_{n}}^{\mathcal{O}}\left(\beta_{1}, \ldots, \beta_{n}\right)
$$

3) Asymptotic behaviour

$$
\mathcal{F}_{\epsilon_{1}, \ldots, \epsilon_{n}}\left(\beta_{1}+\Lambda, \ldots, \beta_{i}+\Lambda, \beta_{i+1}, \ldots, \beta_{n}\right)=O\left(e^{S_{n}^{i}|\Lambda|}\right) \quad \text { for } \quad|\Lambda| \sim \infty \quad
$$

and $S \equiv \max \left(S_{n}^{i}\right)$ is a finite constant.

4) Equation of kinematical poles

$$
\begin{gathered}
-i \lim _{\beta^{\prime} \rightarrow \beta}\left(\beta^{\prime}-\beta\right) \mathcal{F}_{\epsilon \epsilon \epsilon_{1} \ldots \epsilon_{n}}^{\mathcal{O}}\left(\beta^{\prime}+i \pi, \beta, \beta_{1}, \ldots, \beta_{n}\right)= \\
\left(1-e^{2 i \pi\left(w_{\epsilon}+\sum w_{\epsilon_{i} \epsilon}\right)} \prod_{i=1}^{n} S_{\epsilon \epsilon_{i}}\left(\beta-\beta_{i}\right)\right) \mathcal{F}_{\epsilon_{1} \ldots \epsilon_{n}}^{\mathcal{O}}\left(\beta_{1}, \ldots, \beta_{n}\right) .
\end{gathered}
$$

5) Bound state equation: Let particles $A_{i}, A_{j}$ form a bound state $A_{k}$, the corresponding two-particle scattering amplitude exhibits a pole with the residue

$$
-i \lim _{\beta^{\prime} \rightarrow i \epsilon_{\epsilon_{i} \epsilon_{j}}}\left(\beta-i u_{\epsilon_{i} \epsilon_{j}}^{\epsilon_{k}}\right) S_{\epsilon_{i} \epsilon_{j}}(\beta)=\left(\Gamma_{\epsilon_{i} \epsilon_{j}}^{\epsilon_{k}}\right)^{2}
$$

then

$$
\begin{gathered}
-i \lim _{\beta^{\prime} \rightarrow \beta}\left(\beta^{\prime}-\beta\right) \mathcal{F}_{\epsilon_{1} \ldots \epsilon_{i} \epsilon_{j} \ldots \epsilon_{n}}^{\mathcal{O}}\left(\beta_{1}, \ldots, \beta^{\prime}+i \bar{u}_{\epsilon_{i} \epsilon_{k}}^{\epsilon_{j}}, \beta-i \bar{u}_{\epsilon_{j} \epsilon_{k}}^{\epsilon_{i}}, \ldots, \beta_{n-1}\right)= \\
=\Gamma_{\epsilon_{i} \epsilon_{j}}^{\epsilon_{k}} \mathcal{F}_{\epsilon_{1} \ldots \epsilon_{k} \ldots \epsilon_{n}}^{\mathcal{O}}\left(\beta_{1}, \ldots, \beta, \ldots, \beta_{n-1}\right)
\end{gathered}
$$


In [6] it was shown that the operators defined by the form factors (1.2) satisfy proper locality relations provided that they satisfy the properties (1.3)-(1.6) and the residue equations (1.7) and (1.9).

A well established solution method uses the parametrisation of the form factors in terms of the minimal two particle form factor and the explicit prescription of the pole structure. As a first step one determines the minimal two particle form factor, which satisfies Watson's equations for $n=2$,

$$
\mathcal{F}_{a b}^{\min }\left(\beta_{12}\right)=S_{a b}\left(\beta_{12}\right) \mathcal{F}_{b a}^{\min }\left(-\beta_{12}\right), \quad \mathcal{F}_{a b}^{\min }\left(i \pi-\beta_{12}\right)=\mathcal{F}_{b a}^{\min }\left(i \pi+\beta_{12}\right) \quad,
$$

where $\beta_{12}=\beta_{1}-\beta_{2}$. In order to specify an unique solution to these equations one imposes further restrictions, namely that $F^{\text {min }}$ is analytic in $0<\operatorname{Im} \beta<\pi$ and has no zeros in this range. The $n$-particle form factor can then be parametrised as

$$
\begin{gathered}
\mathcal{F}_{\epsilon_{1}, \ldots, \epsilon_{n}}\left(\beta_{1}, \ldots, \beta_{n}\right)= \\
H_{\epsilon_{1}, \ldots, \epsilon_{n}} Q_{\epsilon_{1}, \ldots, \epsilon_{n}}\left(\beta_{1}, \ldots, \beta_{n}\right) \prod_{i<j} \frac{\mathcal{F}_{\epsilon_{i} \epsilon_{j}}^{\min }\left(\beta_{\epsilon_{i} \epsilon_{j}}\right)}{\left(x_{i}+x_{j}\right)^{\delta_{\epsilon_{i} \epsilon_{j}}} \prod_{\alpha}\left(\sinh \frac{1}{2}\left(\beta_{i j}-i \alpha\right) \sinh \frac{1}{2}\left(\beta_{i j}+i \alpha\right)\right)}
\end{gathered}
$$

The product over $\alpha$ runs over all bound state poles deriving from the $S$-matrix; $H_{\epsilon_{1}, \ldots, \epsilon_{n}}$ are normalisation constants, and $x_{i} \equiv e^{\beta_{i}}$.

The only unknowns are therefore the functions $Q_{\epsilon_{1}, \ldots, \epsilon_{n}}\left(x_{1}, \ldots, x_{n}\right)$. They are homogenous functions, analytic apart from the origin and polynomially bounded because of (1.6). They are symmetric functions in their arguments $x_{i}$ and $x_{j}$ if $\epsilon_{i}=\epsilon_{j}$.

Let us focus our attention on the fundamental particle of the theory. In sections $2-4$ we will deal with systems containing only one asymptotic state and therefore only form factors with all indices corresponding to this particle have to be considered. Denote the corresponding function $Q_{11 \ldots 1}=Q_{n}$, for $n$ particles. This is, according to the above discussion, a totally symmetric function. Therefore we introduce the elementary symmetric polynomials, which form a base in the space of symmetric polynomials and are generated by 8

$$
\prod_{i=1}^{n}\left(x+x_{i}\right)=\sum_{k=0}^{n} x^{n-k} \sigma_{k}\left(x_{1}, x_{2}, \ldots, x_{n}\right)
$$


Further we will use the notation that $\bar{x}=1 / x$ and $\bar{\sigma}_{i}\left(x_{1}, \ldots, x_{n}\right)=\sigma_{i}\left(\bar{x}_{1}, \ldots \bar{x}_{n}\right)$. Then the functions $Q_{n}$ can be cast into the following form [[]

$$
Q_{n}\left(x_{1}, \ldots, x_{n}\right)=\sum_{N} \frac{1}{\sigma_{n}^{N-\gamma}} P_{n}\left(x_{1}, \ldots, x_{n}\right)
$$

wherein $\gamma$ denotes a possible non-integer power which is determined from the spin of the corresponding operator through (1.4) and (1.5). The polynomials $P_{n}$ can be expressed as polynomials in the elementary symmetric polynomials $\sigma_{i}$.

Before turning to the explicit construction of solutions of the form factor equations for specific systems, we want to discuss the general structure of the space of local operators. Suppose we have calculated the form factors of a scalar operator (for example the trace of the energy momentum tensor). Representing the operator in terms of its form factors one finds that the form factors of the derivative operators $\partial_{z} \mathcal{O}\left(\partial_{\bar{z}} \mathcal{O}\right)$ are given by $\sigma_{1} \mathcal{F}_{n}$ $\left(\bar{\sigma}_{1} \mathcal{F}_{n}\right)$. This indicates, that in the massive model the two chiral sectors are represented by the variables $x_{i}$ and $\bar{x}_{i}$ respectively. But since

$$
\bar{\sigma}_{i}\left(x_{1}, \ldots, x_{n}\right)=\frac{\sigma_{n-i}\left(x_{1}, \ldots, x_{n}\right)}{\sigma_{n}\left(x_{1}, \ldots, x_{n}\right)}
$$

any $\bar{x}$ dependence can be rewritten in terms of $x$, reflecting the fact that right and left chiral sectors are not independent in the massive theory.

We want to introduce a grading into the space of operators that resembles the structure of highest weight representations of the Virasoro algebra, in order to find their massive counter-parts. It can be seen from (1.11), that increasing the power $N$ in (1.13) for fixed spin $s$, will increase the divergence of the corresponding form factor $\mathcal{F}_{n}$. For that we define as the chiral (left) operators those with the mildest ultraviolet behaviour, which means we put $N=0$ in $(1.13)$. Similarly we conjecture that augmenting $N$ increases the presence of right components in the derivative state. A simple example of this fact is $\bar{L}_{-1} \phi=\bar{\partial} \phi$ as we discussed above. Unfortunately we cannot assign a one-toone correspondence between conformal states $(h, \bar{h})$ and massive states $(N+s, N)$. The parameter $N$ rather indicates how many operators $\bar{L}$ act on the primary operator, but also depends on the explicit representation of $\bar{L}_{n}$ in the massive theory. We will discuss this fact in detail for specific examples. 
Finally we want to stress that setting the scale $\mathrm{N}$ is a rather subtle issue. Because in every module of chiral massive descendent operators, there will be polynomials $P_{n}$ which are proportional to $\sigma_{n}$. Therefore one could identify those as chiral descendents of the primary operator, and regard the original ones as corresponding to $N=1$ states. For some operators this ambiguity can be solved, since one knows the form factors of the primary operators explicitely. But as we will see in section 4 , this scale is not necessarily the same for all primary operators in a theory.

In the next section we analyse the scaling field theory near the critical point of the Ising model, in zero magnetic field, $\mathcal{M}_{3,4}+\phi_{1,3}$. Because of its simple structure (it can be described as a massive free fermion theory), we can introduce our method and test it against known results. In section 3 we investigate the Yang-Lee model, as the simplest example of an interacting field theory. In section 1 we analyse another non-unitary one particle model, $\mathcal{M}_{3,5}+\phi_{1,3}$. Finally, in section 5 we generalise these results to a series of models, namely $\mathcal{M}_{2,2 n+3}+\phi_{1,3}$. As we will see the physical picture persists, while the mathematical methods become more involved.

\section{The Ising Model}

As a first step we will apply the form factor bootstrap to the scaling field theory near the critical point of the two dimensional Ising model in zero magnetic field. This massive field theory can be described in terms of free Majorana fermions, or equally in terms of a bosonic particle which interacts through an $S$-matrix, $S=-1$. The theory is divided into two sectors. One contains the monomials in the fermion fields, and correlation functions of such operators can be calculated by using Wick's theorem. The other sector contains fields which aquire a phase $e^{i \pi}$ when moved around the fermions. The basic fields in this sector are the order and disorder fields $\sigma(x)$ and $\mu(x)$. Their correlation functions have been analysed in numerous ways, and using the form factor approach in [4, 7].

For the scaling Ising model the parametrisation (1.11) takes the explicit form [4, 7, 9]

$$
\mathcal{F}_{n}\left(\beta_{1} \ldots \beta_{n}\right)=Q_{n}\left(x_{1}, \ldots, x_{n}\right) H_{n}\left(\sigma_{n}\right)^{\frac{1}{2} \delta_{w, 0}} \frac{1}{\left(\sigma_{n}\right)^{N}} \prod_{i<j}^{n} \tanh \frac{\left(\beta_{i}-\beta_{j}\right)}{2}
$$


This parametrisation reduces the equation of kinematical poles (1.7) to

$$
\begin{array}{ll}
Q_{n+2}\left(-x, x, x_{1}, \ldots, x_{n}\right)=x^{2 N} Q_{n}\left(x_{1}, \ldots, x_{n}\right), & w=\frac{1}{2}, \\
Q_{n+2}\left(-x, x, x_{1}, \ldots, x_{n}\right)=0, & w=0
\end{array} .
$$

Notice the half-integer power of $\sigma_{n}$ which appears in the sector $w=0$. It has to be introduced in order to satisfy (1.4), and has the effect that form factors with odd index in this sector will carry half integer spin.

The sector $w=\frac{1}{2}$ contains the order and the disorder fields $\sigma$ and $\mu$. The form factors of both operators have been calculated and are given by $Q_{n}=$ const. in (2.1). In [7] the descendent operators of $\sigma(x)$ have been determined. They were constructed by building symmetric polynomials of spin $s$, which are invariant under the recursion relation (2.2). Only invariants with odd spin are consistent with the recursion relation, and can be expressed in terms of the elementary symmetric polynomials as the $n+1$ dimensional determinant [10]

$$
I_{2 n+1}=\left|\begin{array}{ccccc}
\sigma_{1} & \sigma_{3} & \sigma_{5} & \sigma_{7} & \ldots \\
1 & \sigma_{2} & \sigma_{4} & \sigma_{6} & \ldots \\
0 & 1 & \sigma_{2} & \sigma_{4} & \ldots \\
0 & 0 & 1 & \sigma_{2} & \ldots \\
\vdots & \vdots & \vdots & \vdots & \ddots
\end{array}\right|
$$

Arbitrary polynomials formed from the quantities (2.3) constitute solutions to (2.2), which is the equivalent situation as in conformal field theory where the Virasoro irreducible representation of the operator $\phi_{1,2}$ is generated by the application of the operators $L_{-(2 n+1)}$ to the primary field.

The method we want to describe chooses a different basis in the single subspaces of spin $s$. Namely we use the fact that the number of solutions of the recursion relations at level $n$ is given by the number of solutions at level $n-2$ plus the dimension of the kernel of the recursion relations (2.2). This amounts in a simple counting procedure which only involves the comparison of the degree of the polynomials $Q_{n}$ and that of the kernelin. This has the advantage that one finds all solutions to the form factor equations, and not only

\footnotetext{
${ }^{\dagger}$ Note that the second line in (2.2) is precisely the definition of the kernel of the recursion relation.
} 
those which can be considered as descendent operators of some non-trivial primary field. In the case of the Ising model the kernel of the recursion relation (2.2) is given by

$$
\mathcal{K}_{n}=\prod_{i<j}^{n}\left(x_{i}+x_{j}\right)
$$

The total degree of this function is $\operatorname{deg}\left(\mathcal{K}_{n}\right)=\frac{1}{2} n(n-1)$.

Comparing the degree of the polynomial $Q_{n}$ and the degree of $\mathcal{K}_{n}$, we can now construct explicitly solutions of the recursion relations. For the odd form factors, we find the following solutions for the first few values of spin $s$ :

$$
\begin{array}{ll}
s=0 & Q_{1}=\text { const } \\
s=1 & Q_{1}=\sigma_{1} \\
s=2 & Q_{1}=\sigma_{1}^{2} \\
s=3 & Q_{1}=\sigma_{1}^{3}, Q_{3}=\mathcal{K}_{3}
\end{array}
$$

which indicates that we have 1,1,1,2 linear independent solutions at spin levels $s=$ $0,1,2,3$ respectively. Notice that these values correspond exactly to the first terms of the character expansion of the conformal field $\phi_{1,2}$, namely $\tilde{\chi}_{1,2}=1+q+q^{2}+2 q^{3} \ldots$.

We introduce the character $\chi$ of the conformal minimal model as

$$
\chi_{r, s}^{(p, q)}=q^{(h-c / 24)} \tilde{\chi}_{r, s}^{(p, q)}
$$

where $c$ and $h$ denote the central charge and the conformal dimension of the primary operator respectively. Therefore $\tilde{\chi}$ encodes the degeneracies in the Virasoro representation [11],

$$
\tilde{\chi}_{r, s}^{p, q}=\frac{1}{(q)_{\infty}} \sum_{\mu=-\infty}^{\infty}\left(q^{\mu(\mu p q+r q-s p)}-q^{(\mu p+r)(\mu q+s)}\right)
$$

Further, define $\mathcal{P}(m, n)$ to be the number of partitions of $n$ into numbers whose value do not exceed $m$. These partitions are generated by

$$
\frac{1}{(q)_{m}}=\sum \mathcal{P}(m, n) q^{n}
$$

where

$$
(q)_{m} \equiv \prod_{i=1}^{m}\left(1-q^{i}\right)
$$


Using the definition (2.8), we rewrite our counting of the solutions in (2.5) in the following way. At $s=0$ the number of solutions is given by $\mathcal{P}(1,0)$, for $s=1$ by $\mathcal{P}(1,1)$, for $s=2$ by $\mathcal{P}(1,2)$ and for $s=3$ by $\mathcal{P}(1,3)+\mathcal{P}(3,0)$. Using this notation and the generating function (2.9), one immediately finds the number of solutions at general spin $s$, as being generated by

$$
F_{0} \equiv \sum_{m, o d d} \frac{q^{\frac{1}{2} m(m-1)}}{(q)_{m}}=\tilde{\chi}_{1,2}^{(3,4)}(q)
$$

Note that this formalism leads rather to 'fermionic' expressions of the character than to the 'bosonic' form (2.7). This notation ( 'fermionic' and 'bosonic' ) has been introduced by R. Kedem et.al. [28] in their study of character identities for a large class of conformal field theories. Only recently bases have been constructed for conformal field theories which lead to fermionic sum expressions for the characters [15]. It is an interesting fact that the form factor approach leads to them in a straightforward way.

Similarly one can analyse the even form factors, being related to the disorder field $\mu$. The counting procedure can be carried out analogously, and one finds that the number of chiral operators for spin $s$ is generated by

$$
G_{0} \equiv \sum_{m, \text { even }} \frac{q^{\frac{1}{2} m(m-1)}}{(q)_{m}}=\tilde{\chi}_{1,2}^{(3,4)}(q)
$$

As it is well known, both order and disorder fields are related to the conformal operator $\phi_{1,2}$.

As a next step we investigate solutions corresponding to $N=1$ in (2.1). We conjecture that these operators correspond in the ultraviolet limit to states of the type $\bar{L}_{-1} L_{-n_{1}} \ldots L_{-n_{k}}\left|\phi_{1,2}\right\rangle$ (compare figure 1 1 ). Let us repeat the counting as we did for the primary operators. The total degree of the polynomials $Q_{n}$ in this case is given by $\operatorname{deg}\left(Q_{n}\right)=n+s$ which must be compared with the degree of the kernel of the recursion relations.

We list the first few solutions,

$$
\begin{array}{ll}
s=-1 & Q_{1}=\text { const. }, \\
s=0 & Q_{1}=\sigma_{1}, Q_{3}=\mathcal{K}_{3}, \\
s=1 & Q_{1}=\sigma_{1}^{2}, Q_{3}=\sigma_{1} \mathcal{K}_{3} \quad, \\
s=2 & Q_{1}=\sigma_{1}^{3}, Q_{3}=\sigma_{1}^{2} \mathcal{K}_{3}, Q_{3}=\sigma_{2} \mathcal{K}_{3} .
\end{array}
$$




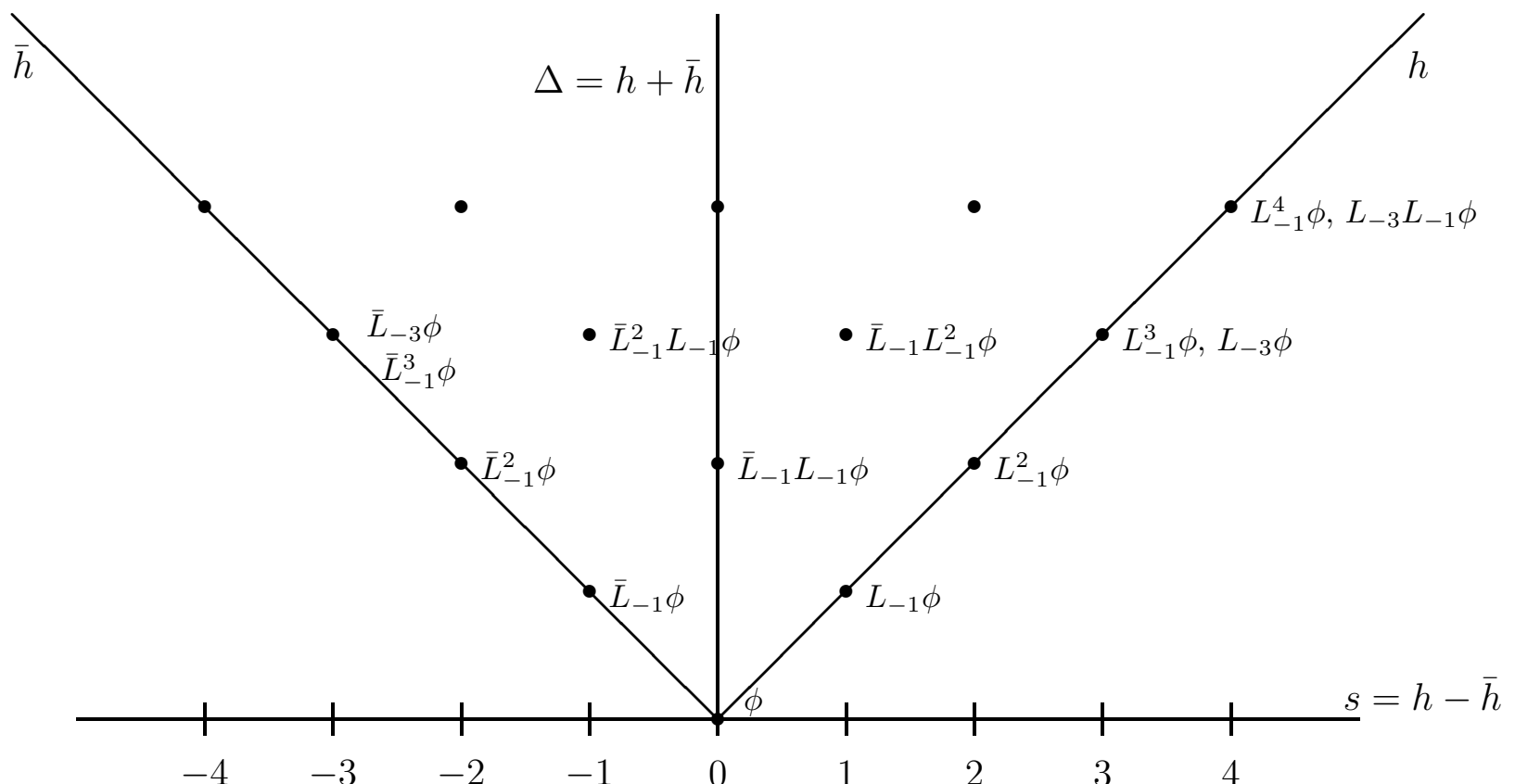

Figure 1: Space of descendent operators of the irreducible Virasoro representation of the field $\phi_{1,2}$ of the conformal Ising model.

The counting here gives not only the derivative states of the type $\bar{L}_{-1} L_{n_{1}} \ldots L_{n_{k}}\left|\phi_{1,2}\right\rangle$ as expected, but also the original chiral ones. For example from (2.1) one immediately realises that the $N=1$ spin zero solution $Q_{1}=\sigma_{1}$ coincides with the $N=0$ spin zero solution $Q_{1}=$ const., which is the one generating the form factors of the primary operator. In the same way every solution $Q_{n}$ being proportional to $\sigma_{n}$ corresponds to an element of the set of chiral descendents.

The generating function of the $N=1$ states can be found in an analogous way as before and is given by

$$
F_{1} \equiv \sum_{m, o d d}^{\infty} \frac{q^{\frac{1}{2} m(m-3)}}{(q)_{m}}
$$

In order to find the level one descendent operators we need to subtract the chiral ones.

$$
\begin{gathered}
F_{1}-F_{0}=\sum_{m, o d d}^{\infty} \frac{q^{\frac{1}{2} m(m-3)}}{(q)_{m}}-\sum_{m, o d d} \frac{q^{\frac{1}{2} m(m-1)}}{(q)_{m}} \\
\sum_{m, \text { odd }}^{\infty} \frac{q^{\frac{1}{2} m(m-3)}\left(1-q^{m}\right)}{(q)_{m}}=\sum_{m, o d d}^{\infty} \frac{q^{\frac{1}{2} m(m-3)}}{(q)_{m-1}}=\sum_{m, \text { even }}^{\infty} \frac{q^{\frac{1}{2}(m+1)(m-2)}}{(q)_{m}}=\bar{q} G_{0}=\bar{q} \tilde{\chi}_{1,2}(q)
\end{gathered}
$$


where we introduced the notation $\bar{q}=q^{-1}$. We have carried out the calculation explicitly, since this re-summation technique is a fundamental tool in the analysis of $q$-sum expressions and will be repeatedly used in the following. We find that in the perturbed model we have the same number of $\bar{h}=1+\frac{1}{16}$ states as in conformal field theory. The analysis of the $N=1$ states in the even sector is in complete analogy, and will therefore be omitted.

Finally let us discuss operators in the subspace $N=2$, again for the odd sector. Generalising from the analysis of the subspace $N=1$ one might expect that in the ultraviolet limit the operators for $N=2$ correspond to states with $\bar{h}=2+\frac{1}{16}$. But for spin $s=-2$ we find two solutions, which we denote by

$$
Q_{1}^{(1)}=\text { const. }, \quad Q_{3}^{(2)}=\sigma_{1} \mathcal{K}_{3},
$$

whereas from conformal field theory we would expect for $\bar{h}=2+\frac{1}{16}, s=-2$ only one operator (compare figure 11). We conclude that the simple interpretation of $N \sim \bar{h}$ does not hold in general. The further we increase $N$, the more terms the specific subspace will contain (e.g. for $N=3, s=-3$ we find 6 operators). This fact can be understood from the explicit representation of the invariants (2.3). They have partial degree $d_{p}\left(I_{2 n+1}\right)=$ $n+1$, or specifically for our example from above $d_{p}\left(I_{3}\right)=2$. This means we can write $I_{3} / \sigma_{3}^{2}=\bar{I}_{3}$, where $\bar{I}_{n}$ denotes the same invariants (2.3) but in the variables $\bar{x}$. Using these expressions the states $(2.13)$ can be related to conformal states as

$$
Q^{(1)} \sim \bar{L}_{-1}^{2}, \quad Q^{(2)} \sim \bar{L}_{-3} L_{-1}
$$

Similar for all spins $s$ in the subspace $N=2$ we expect states of the kind $\bar{L}_{-3} L_{-n_{1}} \ldots L_{-n_{k}}\left|\phi_{1,2}\right\rangle$. This is indeed the case, since the generating function for the $N=2$ solutions is given by

$$
F_{2}=\sum_{m, o d d} \frac{q^{\frac{1}{2} m(m-1)-2 m}}{(q)_{m}}=\left(1+\bar{q}+\bar{q}^{2}+\bar{q}^{3}\right) \tilde{\chi}_{1,2}(q)
$$

that is, it resembles exactly the structure of $\tilde{\chi}_{1,2}(\bar{q}) \tilde{\chi}_{1,2}(q)$ up to second order in $\bar{q}$, but additionally contains one term $\bar{q}^{3} \tilde{\chi}_{1,2}(q)$.

The presence of such higher level operators makes a direct link between the parameter $N$ and the dimensions of the conformal fields difficult. Nevertheless increasing $N$ we can 
gradually build up the whole space of operators in the massive theory. The number of operators at level $N$ is generated by

$$
F_{N} \equiv \sum_{m=1, o d d}^{\infty} \frac{q^{\frac{1}{2} m(m-1)-N m}}{(q)_{m}}
$$

in the odd sector, and by

$$
G_{N} \equiv \sum_{m=0, \text { even }}^{\infty} \frac{q^{\frac{1}{2} m(m-1)-N m}}{(q)_{m}}
$$

in the even sector. These functions satisfy the recursion relations

$$
F_{N}=F_{N-1}-\bar{q}^{N} G_{N-1}, \quad G_{N}=G_{N-1}+\bar{q}^{N} F_{N-1}
$$

with the initial conditions $G_{0}=F_{0}=\tilde{\chi}_{1,2}(q)$. Because of the symmetry of the recursion relations and the initial conditions, it follows that $F_{N}=G_{N}$. This implies that $F_{N}=$ $\left(1+q^{N}\right) F_{N-1}$ which can be solved to give $F_{N}=G_{N}=\prod_{k=1}^{N}\left(1+\bar{q}^{k}\right) F_{0}$, which resembles the character expansion of $\tilde{\chi}_{1,2}(\bar{q}) F_{0}$ up to order $N$. Note that the whole space of states is generated by $\lim _{N \rightarrow \infty} F_{N}$. This can be written as

$$
F_{\infty}=\tilde{\chi}_{1,2}(q) \tilde{\chi}_{1,2}(\bar{q})
$$

and similarly for $G_{\infty}$.

A few comments follow, in order to clarify the meaning of these expressions. We find that $F_{\infty}$ and $G_{\infty}$ generate the space of operators in this sector. They have the identical form of the conformal expression of the space of descendent operators of the primary field $\phi_{1,2}$. Further note that in (2.15) the characters of the two chiralities are separated. This is a main feature of conformal field theories, where the right and left movers can be treated independently. Here in the massive theory the two chiralities are present, but $q$ and $\bar{q}$ are not independent variables, and therefore such a factorisation can only be regarded as a formal expression.

Finally we want to stress the fact that one obtains for finite $N$ some 'finitized' expressions for the Virasoro characters. In this approach they appear naturally in the counting procedure of states. Note that the same expressions turn up in the study of corner transfer matrices where the finitization is related to the fact that one has a discrete system of 
finite size (see e.g. [16]). It would be interesting to understand whether there is a deeper reason for this relation.

The sector $w=0$ has been discussed in [17]. In this sector the kinematical recursion relation maps the form factors onto zero, $\mathcal{F}_{n}\left(\beta+i \pi, \beta, \beta_{1}, \ldots, \beta_{n}\right)=0$. It follows that form factors with different particle number $n$ are not linked, and therefore any kernel solution represents an acceptable form factor from the point of view of the form factor equations. The analysis can be carried out in a similar way as for the sector $w=\frac{1}{2}$. The result is that the even form factors generate the descendent spaces of the energy operator, which scales in the ultraviolet limit as $\left(\frac{1}{2}, \frac{1}{2}\right)$ and of the identity operator $(0,0)$. The odd

form factors generate the spaces of the fermions $\left(\frac{1}{2}, 0\right)$ and $\left(0, \frac{1}{2}\right)$. The ultraviolet limits of the correlation functions can be calculated explicitly for these operators and the scaling dimensions coincide with the corresponding conformal operators.

The thermal Ising model has been examined also by various other means. But, since it can be described as a free field theory, many methods and results remain confined to this special model. The interesting fact about the method we have presented in this chapter is that it can be straightforwardly applied also to interacting theories. In fact any massive integrable field theory having a description in terms of a scattering matrix can be investigated in this way. We will give several examples in the following sections.

\section{Space of Operators in the massive Yang-Lee model}

The scaling Yang-Lee model can be described as a massive integrable field theory 18. Its on-mass-shell spectrum consists of a single massive scalar particle of mass $m$. Also the conformal field theory $\mathcal{M}_{2,5}$ is quite simple containing only one primary field $h=-\frac{1}{5}$ besides the identity operator.

The scattering matrix of the massive particles is determined by the following twoparticle amplitude 18

$$
S(\beta) \equiv \frac{\tanh \frac{1}{2}(\beta+i \alpha)}{\tanh \frac{1}{2}(\beta-i \alpha)}
$$

with $\alpha=\frac{2}{3} \pi$ f.

\footnotetext{
$\ddagger$ We keep whenever possible the notation general since we can use then some of the results we will
} 
The form factors have been analysed in [10, 19, 20]. By defining the minimal two particle form factor as

$$
\mathcal{F}_{11}^{\min }(\beta)=(-i) \sinh \frac{\beta}{2} \exp \left\{\int_{0}^{\infty} \frac{d x}{x} \frac{\cosh x\left(\frac{1}{2}-\frac{\alpha}{\pi}\right)}{\cosh \frac{x}{2}} \frac{\sin ^{2} \frac{x(i \pi-\beta)}{2 \pi}}{\sinh x}\right\},
$$

the parametrisation (1.11) of the form factors reads for this model as

$\mathcal{F}_{n}\left(\beta_{1}, \ldots, \beta_{n}\right)=H_{n} Q_{n}\left(\beta_{1}, \ldots, \beta_{n}\right) \prod_{i<j} \frac{\mathcal{F}^{\min }\left(\beta_{i}-\beta_{j}\right)}{\left(x_{i}+x_{j}\right) \sinh \frac{1}{2}\left(\beta_{i}-\beta_{j}-i \alpha\right) \sinh \frac{1}{2}\left(\beta_{i}-\beta_{j}+i \alpha\right)}$,

where $Q_{n}$ is of the form (1.13). The constants $H_{n}$ in (3.3) are given by

$$
H_{n}=C_{0}\left(4 \cos ^{2} \frac{\alpha}{2} \sin \alpha\right)^{\frac{n}{2}}(\zeta(i \pi) \pi)^{\frac{(n-1)^{2}-1}{2}} i^{n^{2}}
$$

The form factor equations have been reduced through this parametrisation to two coupled recursive relations for $Q_{n}$ [20], namely

$$
Q_{n+2}\left(-x, x, x_{1}, \ldots, x_{n}\right)=D_{n}\left(x, x_{1}, \ldots, x_{n}\right) Q_{n}\left(x_{1}, \ldots, x_{n}\right)
$$

with the recursion function

$$
D_{n}\left(x, x_{1}, \ldots, x_{n}\right)=\sum_{k=1}^{n} \sum_{m=1, \text { odd }}^{k}[m] x^{2(n-k)+m} \sigma_{k}^{(n)} \sigma_{k-m}^{(n)}(-1)^{k+1},
$$

wherein the symbol $[l]$ is defined by

$$
[l] \equiv \frac{\sin (l \alpha)}{\sin \alpha}
$$

The second recursion relation derives from the bound state axiom (1.9) and reads as

$$
Q_{n+1}\left(x \omega^{\frac{1}{2}}, x \omega^{-\frac{1}{2}}, x_{1}, \ldots, x_{n-1}\right)=x \prod_{i=1}^{n-1}\left(x+x_{i}\right) Q_{n}\left(x, x_{1}, \ldots, x_{n-1}\right)
$$

The recursion relation (3.5) has been studied in [21] for the primary massive operators. It admits an infinite number of solutions. A basis in the solution space is given by the polynomials

$$
Q_{n}=\mathcal{Q}_{n}(k) \equiv\left\|M_{i j}(k)\right\|, \quad k \in \mathbf{Z} \quad,
$$

obtain here for the analysis of the form factors of the models $\mathcal{M}_{2,2 n+3}$, which will be discussed in section 5. This is due to the fact that the $S$-matrix of the fundamental particle of these theories is given by (3.1) with $\alpha=\frac{2}{2 n+1} \pi$. 
where $M_{i j}(k)$ is an $(n-1) \times(n-1)$ matrix whose entries are

$$
M_{i j}(k)=\sigma_{2 i-j}[i-j+k] \quad .
$$

Enforcing in addition to (3.5) also the bound state recursion relation (3.8) only one consistent solution exists, which is given by $\mathcal{Q}_{n}(1)$ [22]. In the ultraviolet limit the operator corresponding to this solution scales as the conformal primary operator $\phi_{1,2}$ of the minimal model $\mathcal{M}_{2,5}$ [20]. For $w=\frac{1}{2}$ the equation (3.5) admits only one single solution [24], which is not consistent with the bound state equation (3.8).

We are interested in the analysis of the space of descendent operators of this model. From (1.13) we find that the functions $Q_{n}$ take the form $Q_{n}\left(x_{1}, \ldots, x_{n}\right)=\sigma_{n}^{-N} P_{n}\left(x_{1}, \ldots, x_{n}\right)$. We start by analysing the space of chiral descendents, $N=0$. Note, that in this model even and odd form factors are coupled due to the bound state equation (3.8). The method is in principle the same as in the Ising model. We solve the relations (3.5) and (3.8) recursively using the property that the space of solutions at level $n$ is given by the number of solutions at level $n-1$ plus the dimension of the kernel of the combined recursion relations. In table 11 we have written down the degree for the polynomials $P_{n}$ required from Lorentz invariance and the dimension of the kernel of the recursion relations.

Let us now count the independent solutions for various spin levels. For $s=1$ the only solution is that generated by $P_{1}=\sigma_{1}$, which corresponds exactly to the level 1 descendent of the primary field $\phi$ of the Yang-Lee model. For spin 2 we have two possible solutions, generated by the polynomials

$$
P_{1}=\sigma_{1}^{2}, \quad P_{2}=\mathcal{K}_{2}
$$

Similarly higher spin values can be investigated. From table 1 we find that a kernel solution for $n=3$ occurs only for $\operatorname{spin} s \geq 6$. Let us examine the solution space for the particular spin value $s=6$. The solutions are

$$
\begin{aligned}
& P_{1}=\sigma_{1}^{6} \\
& P_{2}=\sigma_{1}^{4} \mathcal{K}_{2}, \sigma_{2} \sigma_{1}^{2} \mathcal{K}_{2}, \sigma_{2}^{2} \mathcal{K}_{2} \\
& P_{3}=\mathcal{K}_{3}
\end{aligned}
$$

that is we have 5 independent operators. 
We compare these values with the dimensions of the spaces of descendent operators in conformal field theory. The number of descendent operators is given by the character expansions,

\begin{tabular}{||c|c|c||}
\hline$n$ & $\operatorname{deg}\left(Q_{n}^{(s)}\right)=\frac{1}{2} n(n-1)+s$ & $\operatorname{deg} \mathcal{K}=\frac{3}{2} n(n-1) n \geq 2$ \\
\hline 1 & $0+s$ & 0 \\
2 & $1+s$ & 3 \\
3 & $3+s$ & 9 \\
4 & $6+s$ & 18 \\
5 & $10+s$ & 30 \\
6 & $15+s$ & 45 \\
7 & $21+s$ & 63 \\
\hline
\end{tabular}

Table 1: Comparing the total degrees of the polynomials $P_{n}$ for the case $N=0$ with the kernel of the recursion relations for spin $s$ and level $n$.

$$
\begin{aligned}
& \chi_{1,1}=1+q^{2}+q^{3}+q^{4}+2 q^{5}+2 q^{6}+O\left(q^{7}\right) \\
& \chi_{1,3}=1+q+q^{2}+q^{3}+2 q^{4}+2 q^{5}+3 q^{6}+O\left(q^{7}\right)
\end{aligned}
$$

Summing up these values we find that the Yang-Lee CFT contains 1 descendent operator at spin 1, 2 operators at spin 2 and 5 operators at spin 6 . We compare these values with the ones obtained in the perturbed model and find that they coincide.

In table 2 we have collected the number of independent solutions of the recursion relations using the notation of partitions introduced in (2.8). The counting can now be generalised to arbitrary values of $s$. A new kernel solution will contribute, whenever $s=n(n-1)$. Therefore the number of solutions is generated by

$$
F_{1}=\sum_{n=0}^{\infty} \frac{q^{n(n-1)}}{(q)_{n}}
$$

where we introduce the general definition,

$$
F_{N} \equiv \sum_{n=0}^{\infty} \frac{q^{n^{2}-N n}}{(q)_{n}}
$$




\begin{tabular}{||l|l||}
\hline$s=0$ & $\mathcal{P}(1,0)$ \\
$s=1$ & $\mathcal{P}(1,1)$ \\
$s=2$ & $\mathcal{P}(1,2)+\mathcal{P}(2,0)$ \\
$s=3$ & $\mathcal{P}(1,3)+\mathcal{P}(2,1)$ \\
& $\vdots$ \\
$s=6$ & $\mathcal{P}(1,6)+\mathcal{P}(2,4)+\mathcal{P}(3,0)$ \\
\hline
\end{tabular}

Table 2: Number of independent solutions of the recursion equations for several spin values $s$.

For the Yang-Lee model the Rogers-Ramanujan identities (see e.g. [12, 13]) relate the so-called [28] 'fermionic' sum expressions to the 'bosonic' form of [11, 10] as

$$
\begin{aligned}
& \tilde{\chi}_{1,1}^{(2,5)}(q)=\prod_{i=0}^{\infty} \frac{1}{\left(1-q^{2+5 i}\right)\left(1-q^{3+5 i}\right)}=F_{-1}, \\
& \tilde{\chi}_{1,2}^{(2,5)}(q)=\prod_{i=0}^{\infty} \frac{1}{\left(1-q^{1+5 i}\right)\left(1-q^{4+5 i}\right)}=F_{0} .
\end{aligned}
$$

By a re-summation one can show that

$$
F_{1}=F_{0}+F_{-1}=\tilde{\chi}_{1,1}(q)+\tilde{\chi}_{1,2}(q)
$$

which means that the number of chiral descendent operators at each level is the same in the conformal and the perturbed Yang-Lee model. It is interesting to note that we have found the sum of the two characters, and not the two Virasoro irreducible representations modules separately. This is expected from the physical point of view, since in the perturbed model a mixing of the two spaces occurs. This has been analysed by Zamolodchikov [1], by determining the conservation laws of the perturbed theory. The simplest such conservation law, is given by

$$
\partial_{\bar{z}} T \sim \partial_{z} \phi_{1,2}
$$

where $T$ denotes the holomorphic component of the energy momentum tensor, which is an element of the Virasoro irreducible representation of the identity operator in the conformal model. This relation expresses the continuity equation for the stress energy tensor in the massive model. 
Next we investigate the solutions corresponding to $Q_{n}=\frac{1}{\sigma_{n}} P_{n}$, i.e. $N=1$ in (1.13). Let us repeat the counting as we have done for the primary operators. The total degree of the polynomials $P_{n}$ is now given by $\operatorname{deg}\left(P_{n}\right)=\frac{1}{2} n(n-1)+n+s$. The first few solutions are

$$
\begin{array}{ll}
s=-1 & P_{1}=\text { const. }, \\
s=0 & P_{1}=\sigma_{1}, P_{2}=\mathcal{K}_{2} \quad, \\
s=1 & P_{1}=\sigma_{1}^{2}, P_{2}=\sigma_{1} \mathcal{K}_{2} \quad, \\
s=2 & P_{1}=\sigma_{1}^{3}, P_{2}=\sigma_{1}^{2} \mathcal{K}_{2}, P_{2}=\sigma_{2} \mathcal{K}_{2} \quad .
\end{array}
$$

As in the Ising model this amounts in an over-counting of the states, since the chiral operators appear in the subspace $N=1$ as solutions proportional to $\sigma_{n}$, i.e. for which $P_{n} \sim \sigma_{n}$

The generating function of the $N=1$ states is given by $F_{2}$. In order to find the structure of this subspace, we subtract the chiral operators using the $q$-sum relation

$$
F_{2}-F_{1}=\bar{q} F_{0}=\bar{q} \tilde{\chi}_{13}(q)
$$

The structure of the $N=1$ space coincides with that of the descendent operators of the conformal scalar primary fields $\phi_{1,1}$ and $\phi_{1,3}$ for $\bar{h}=1$. In CFT there are only states proportional to $\tilde{\chi}_{1,3}(q)$ since $\bar{L}_{-1}|I\rangle=0$. We find therefore that the subspace $N=1$ in the massive model corresponds to the space $\bar{h}=1+\bar{h}_{0}$ of the conformal model, where $h_{0}$ stands for the right chiral dimension of the conformal primary fields.

Using the generating function (3.14) one can show that the number of operators in the subspace $N$, is generated by $F_{N+1}$. This function satisfies the recursion identity

$$
F_{N}=F_{N-1}+\bar{q}^{N-1} F_{N-2}
$$

with the initial condition of $F_{0}=\tilde{\chi}_{1,3}(q)$ and $F_{-1}=\tilde{\chi}_{1,1}(q)$. It has been shown by I. Schur (see e.g. [13]) that the solution to this problem is given by

$$
F_{N}=D_{1}^{(N-1)}(\bar{q}) \tilde{\chi}_{1,3}(q)+D_{2}^{(N-2)}(\bar{q}) \tilde{\chi}_{1,1}
$$

with

$$
D_{1}^{(N)}(\bar{q})=\sum_{\mu=-\infty}^{\infty}(-1)^{\mu} \bar{q}^{\frac{\mu}{2}(5 \mu+1)}\left[\begin{array}{c}
N+1 \\
{\left[\frac{N+1-5 \mu}{2}\right]}
\end{array}\right]
$$




$$
D_{2}^{(N)}(\bar{q})=\sum_{\mu=-\infty}^{\infty}(-1)^{\mu} \bar{q}^{\frac{\mu}{2}(5 \mu-3)}\left[\begin{array}{c}
N+1 \\
1+\left[\frac{N+1-5 \mu}{2}\right]
\end{array}\right]
$$

which are finitized forms of the characters $\tilde{\chi}_{1,3}(\bar{q})$ and $\tilde{\chi}_{1,1}(\bar{q})$ respectively. From the recursion relation $(3.17)$ it follows that $F_{N}=F_{N-1}\left(\bmod \bar{q}^{N}\right)$, and therefore we find that $F_{N}$ resembles the partition function of the Yang-Lee model up to order $\bar{q}^{N}$. Similarly as in the Ising model we can write the formal identity

$$
\lim _{N \rightarrow \infty} F(N)=Z_{2,5}=\tilde{\chi}_{1,3}(q) \tilde{\chi}_{1,3}(\bar{q})+\tilde{\chi}_{1,1}(q) \tilde{\chi}_{1,1}(\bar{q})
$$

There are several new features in this model with respect to the Ising model. First in the form factor approach we cannot disentangle members of the Virasoro irreducible representations of the identity operator and the primary field. As we have explained this is due to the fact, that a mixing of these Virasoro irreducible representations occurs in the massive theory. On the other hand if the structure of the spaces are isomorphic one should be able to find a unique mapping of the operators.

Actually a similar structure appears in the Ising model in the sector $w=0$, which contains the descendent operators of the massive scalar primary fields $\phi_{1,3}$ and $\phi_{1,1}$. Also there the same argument of the mixing of operators holds. The special fact in the Ising model is that for the sector $w=0$ the UV dimensions of the operators can be calculated explicitly. Therefore it is possible to assign in a unique manner the form factors to specific conformal operators. It has been shown in [17] that in the Ising model $(w=0)$ chiral operators in the descendent space of the identity operator have form factors which are proportional to $\sigma_{n}$ while the others form the chiral descendents of the primary field $\phi_{1,3}$.

It is tempting to conjecture a similar structure in the Yang-Lee model. This is due to the fact that the space of solutions with $P_{n} \nsim \sigma_{n}$ are given by

$$
\sum_{n} \frac{q^{n(n-1)}}{(q)_{n-1}}=\tilde{\chi}_{1,1}(q)
$$

This seems to be the opposite situation as in the Ising model, but one has to recall that in the Yang-Lee model the field $\phi_{1,3}$ has negative scaling dimension, and therefore behaves less singular as the identity operator in the UV limit. Unfortunately we are not able to calculate the UV dimensions exactly in this model, and therefore this conjecture cannot be confirmed. 
Finally we want to discuss the relation of the space of operators to the conserved charges of the model. In [10] P. Christe has constructed descendent operators of the primary field. His approach was to modify the construction of [7] for the Ising model to the case of the Yang-Lee model, i.e. he constructed polynomial invariants which satisfied both recursion relations (3.5) and (3.8). He found invariants of spin values $s=1,5 \bmod (6)$. They correspond to the infinite set of integrals of motions of the massive theory. Note that in the Ising model polynomials formed the corresponding invariants can be mapped one-to-one to the fields of the conformal Virasoro irreducible representation. Obviously this is not the case for the Yang-Lee model, since the operators of the Virasoro irreducible representation of $\phi_{1,2}$ are generated by $L_{5 n+1}, L_{5 n+4}$. Constructing derivative operators through polynomial invariants one only finds a sub-space of the descendents of the conformal operator $\phi_{1,2}$. This implies that the conserved charges do not generate the whole space of operators. This feature generalises to all further models we will consider. The Ising model is an exception since it can be considered as a free field theory.

\section{The model $\mathcal{M}_{3,5}$}

As a further simple model whose on-shell spectrum contains only one particle is the $\phi_{1,3}$ perturbation of $\mathcal{M}_{3,5}$. Let us recall the basic properties of the conformal model. It is a non-unitary minimal model, with the primary operators given through the Kac table

\begin{tabular}{|c|c|}
\hline$\frac{3}{4}$ & 0 \\
\hline$\frac{1}{5}$ & $-\frac{1}{20}$ \\
\hline$-\frac{1}{20}$ & $\frac{1}{5}$ \\
\hline 0 & $\frac{3}{4}$ \\
\hline
\end{tabular}

The characters of these operators are [11, 28],

$$
\begin{aligned}
& \tilde{\chi}_{1,1}(q)=\sum_{n=0, \text { even }} \frac{q^{\frac{n(n+2)}{4}}}{(q)_{n}}=\sum_{\mu=-\infty}^{\infty} q^{\mu(15 \mu+2)}-q^{(3 \mu+1)(5 \mu+1)}, \\
& \tilde{\chi}_{1,2}(q)=\sum_{n=0, \text { even }} \frac{q^{\frac{n^{2}}{4}}}{(q)_{n}}=\sum_{\mu=-\infty}^{\infty} q^{\mu(15 \mu-1)}-q^{(3 \mu+1)(5 \mu+2)}
\end{aligned}
$$




$$
\begin{aligned}
& \tilde{\chi}_{1,3}(q)=\sum_{n=0, \text { odd }} \frac{q^{\frac{n^{2}-1}{4}}}{(q)_{n}}=\sum_{\mu=-\infty}^{\infty} q^{\mu(15 \mu-4)}-q^{(3 \mu+1)(5 \mu+3)} \\
& \tilde{\chi}_{1,4}(q)=\sum_{n=0, \text { odd }} \frac{q^{\frac{n(n+2)-3}{4}}}{(q)_{n}}=\sum_{\mu=-\infty}^{\infty} q^{\mu(15 \mu-7)}-q^{(3 \mu+1)(5 \mu+4)} .
\end{aligned}
$$

We have collected the bosonic and the fermionic expressions for later convenience.

Perturbing the conformal model in the direction of the $\phi_{1,3}$ operator one obtains a massive field theory, containing one asymptotic particle without a bound state. The interaction of the asymptotic states is given through the $S$-matrix 26]

$$
S(\beta)=-i \tanh \frac{1}{2}\left(\beta-i \frac{\pi}{2}\right) \quad .
$$

An interesting novel fact with respect to the previous models is, that these particles have non trivial statistics, since $\lim _{\beta \rightarrow \pm \infty} S(\beta)=\mp i$, and are in fact related to the operator $\phi_{2,1}=\phi_{1,4}$ of the model [27]. Because they are spin $\frac{1}{4}$ particles they also have $w_{i j}=\mp \frac{1}{4}$ depending which chirality of the operator we choose (or equivalently if we define $w_{i j}$ through $\pm \infty$ in rapidity space).

The minimal two particle form factor can be determined as 25]

$$
\mathcal{F}^{\min }(\beta)=\sinh \frac{\beta}{2} \prod_{k=0}^{\infty} \frac{\Gamma\left(k+\frac{1}{4}-i \frac{\beta}{2 \pi}\right) \Gamma\left(k+\frac{5}{4}+i \frac{\beta}{2 \pi}\right)}{\Gamma\left(k+\frac{3}{4}-i \frac{\beta}{2 \pi}\right) \Gamma\left(k+\frac{7}{4}+i \frac{\beta}{2 \pi}\right)} \quad .
$$

Since there are no bound states the parameterisation of the form factor is given by

$$
\mathcal{F}_{n}\left(\beta_{1}, \ldots, \beta_{n}\right)=H_{n} Q_{n}\left(x_{1}, \ldots, x_{n}\right) \sigma_{n}^{\frac{n}{4}} \sigma_{n}^{-\frac{1}{2} \delta_{w, 0}} \prod_{i<j} \frac{\mathcal{F}^{\min }\left(\beta_{i j}\right)}{x_{i}+x_{j}}
$$

where we define the normalisation constants $H_{n}$ such that they satisfy

$$
H_{n+2}=\left(\frac{2 i}{\pi}\right)^{n} \frac{1}{\mathcal{F}^{\min }(i \pi)} H_{n}
$$

The fractional power of $\sigma_{n}$ in (4.4) in the sector $w=0$ is needed in order to satisfy (1.4). As a consequence we will find that operators corresponding to odd form factors will have non-zero spin, namely the same as the asymptotic particles, $s= \pm \frac{1}{4}$.

Using the parametrisation (4.4) and taking into account the statistics of the asymptotic states, the recursion relation (1.7) reduces to

$$
Q_{n+2}\left(-x, x, x_{1}, \ldots, x_{2 n}\right)=(-x)^{\delta_{w, 0}} D_{n}\left(x, x_{1}, \ldots, x_{n}\right) Q_{n}\left(x_{1}, \ldots, x_{2 n}\right)
$$


where

$$
D_{n}\left(x, x_{1}, \ldots, x_{n}\right)=\sum_{k=0}^{n}\left(e^{(n-k) \frac{i \pi}{2}}-e^{2 i \pi w} e^{-(n-k) \frac{i \pi}{2}}\right) x^{n-k} \sigma_{k}^{n} \quad .
$$

We first analyse the sector $w=0$. Even and odd form factors are not linked by the recursive equations, and we start by examining the even ones. As before we use the parametrisation (1.13) to introduce a grading in the space of operators. We have $\gamma=0$ since the fractional powers of $\sigma_{n}$ have already been taken in account in (4.4).

Since we have carried out the counting technique already in detail for the Ising and the Yang-Lee model we will merely state the results. For chiral operators $(N=0)$ the degree of $P_{n}$ is given by $\operatorname{deg}\left(P_{n}\right)=\frac{n^{2}}{4}+s$. For spin $s=0$ there exists only one solution which was found in [25], and corresponds to the perturbation of the scalar primary field $\phi_{1,3}$. Its form factors are given by $Q_{n}=\left\|\sigma_{4 i-2 j-1}\right\|, n=2,4, \ldots$ in (4.4), where the dimension of this determinant is $\frac{n}{2}$.

For spin $s=0,1,2$ one finds $1,1,3$ solutions respectively, while for general spin values the generating function for the number of independent solutions is

$$
F_{1}^{e}=\sum_{n, \text { even }} \frac{q^{\frac{n(n-2)}{4}}}{(q)_{n}} .
$$

By shifting the summation index and using the fermionic sum expressions of the characters (4.2) it is not difficult to show that

$$
F_{1}^{e}=\tilde{\chi}_{1,1}(q)+\tilde{\chi}_{1,3}(q)
$$

We want to analyse the full space of operators, and therefore increase the value $N$ in (1.13) by integer powers. The generating function for states at level $N$ is given by

$$
F_{N}^{e} \equiv \sum_{n, \text { even }} \frac{q^{\frac{n(n+2)}{4}-N n}}{(q)_{n}}
$$

Let us further define

$$
G_{N}^{o} \equiv \sum_{n, o d d} \frac{q^{\frac{n^{2}-1}{4}-N n}}{(q)_{n}}
$$

then these functions satisfy the recursion identities

$$
\begin{aligned}
& F_{N}^{e}=F_{N-1}^{e}+\bar{q}^{N-1} G_{N-1}^{o}, \\
& G_{N}^{o}=G_{N-1}^{o}+\bar{q}^{N} F_{N}^{e} .
\end{aligned}
$$


In order to separate formally the chiral sectors, we express these infinite sums in terms of the characters. From the recursion relations it is clear that $F_{N}^{e}$ and $G_{N}^{o}$ can be written as polynomials in $\bar{q}$ multiplied by the characters, which are the initial conditions of the recursion relations, namely $F_{0}^{e}=\tilde{\chi}_{1,1}(q)$ and $G_{0}^{o}=\tilde{\chi}_{1,3}(q)$. Expanding for the first few values of $N$ one finds indeed that

$$
F_{N}^{e}=\tilde{\chi}_{1,1}(q) \tilde{\chi}_{1,1}(\bar{q})+\tilde{\chi}_{1,3}(q) \tilde{\chi}_{1,3}(\bar{q})\left(\bmod \bar{q}^{N}\right)
$$

The general solution of the recursion relations can be cast into the form

$$
\begin{aligned}
& F_{N}^{e}=D_{1}^{(N)}(\bar{q}) \tilde{\chi}_{1,1}(q)+D_{3}^{(N)}(\bar{q}) \tilde{\chi}_{1,3}(q) \\
& G_{N}^{o}=D_{4}^{(N)}(\bar{q}) \tilde{\chi}_{1,1}(q)+D_{2}^{(N)}(\bar{q}) \tilde{\chi}_{1,3}(q)
\end{aligned}
$$

with

$$
\begin{aligned}
& D_{1}^{(N)}(\bar{q})=\sum_{\mu=-\infty}^{\infty} \bar{q}^{\mu(15 \mu+2)}\left[\begin{array}{c}
2 N \\
N-5 \mu
\end{array}\right]-\bar{q}^{(3 \mu+1)(5 \mu+1)}\left[\begin{array}{c}
2 N \\
N-1-5 \mu
\end{array}\right] \rightarrow \tilde{\chi}_{1,1}(\bar{q}), \\
& D_{2}^{(N)}(\bar{q})=\sum_{\mu=-\infty}^{\infty} \bar{q}^{\mu(15 \mu+1)}\left[\begin{array}{c}
2 N \\
N-5 \mu
\end{array}\right]-\bar{q}^{(3 \mu+1)(5 \mu+2)}\left[\begin{array}{c}
2 N \\
N-3-5 \mu
\end{array}\right] \rightarrow \tilde{\chi}_{1,2}(\bar{q}), \\
& D_{3}^{(N)}(\bar{q})=\sum_{\mu=-\infty}^{\infty} \bar{q}^{\mu(15 \mu-4)}\left[\begin{array}{c}
2 N \\
N-1-5 \mu
\end{array}\right]-\bar{q}^{(3 \mu+1)(5 \mu+3)}\left[\begin{array}{c}
2 N \\
N-2-5 \mu
\end{array}\right] \rightarrow \tilde{\chi}_{1,3}(\bar{q}), \\
& D_{4}^{(N)}(\bar{q})=\sum_{\mu=-\infty}^{\infty} \bar{q}^{\mu(15 \mu+7 \mu+1)}\left[\begin{array}{c}
2 N \\
2 N \\
N-1-5 \mu
\end{array}\right]-\bar{q}^{(3 \mu+2)(5 \mu+1)+1}\left[\begin{array}{c}
2-5 \mu
\end{array}\right] \rightarrow \bar{q} \tilde{\chi}_{1,4}(\bar{q})
\end{aligned}
$$

where the limits are understood as $N \rightarrow \infty$.

While $F_{N}^{e}$ generates the even part of the partition function as we expect, it seems that the function $G_{N}^{o}$ lacks a physical interpretation in this context. Its importance will be clarified in the following.

Let us introduce a compact notation in order to identify the operators corresponding to a specific set of form factors. If the space of operators in a certain sector can be decomposed as $\tilde{\chi}_{r, s}(q) \tilde{\chi}_{r^{\prime}, s^{\prime}}(\bar{q})$ we will call the corresponding chiral (i.e. with the lowest possible divergence of the form factors) operator with spin $s=h_{r, s}-h_{r^{\prime}, s^{\prime}}$, as the perturbed primary field $\left(h_{r, s}, h_{r^{\prime}, s^{\prime}}\right)$. In this notation the space of even form factors in the sector $w=0$ contains the descendents of the primary fields $\left(\frac{1}{5}, \frac{1}{5}\right)$ and $(0,0)$. 
The analysis of the even form factors is complete, and we turn to the odd sector. From (1.5) and (4.4) we find that it will contain particles of spin $-\frac{1}{4}+\mathbf{Z}$. By applying the counting method we find that the space of chiral descendents is given by

$$
F_{1}^{o} \sum_{n, \text { odd }} \frac{q^{\frac{n(n-2)}{4}}}{(q)_{n}}=q^{-\frac{1}{4}} \tilde{\chi}_{1,2}(q)+q^{\frac{3}{4}} \tilde{\chi}_{1,4}(q)
$$

which indicates that $\phi_{1,4}$ does not mix with the primary operator $\phi_{1,2}$ but with its descendent at level one. Compare this situation with the table of conformal dimensions of the model (4.1). The dimension of the field $\phi_{1,4}\left(h=\frac{3}{4}\right)$ is by far 'closer' to that of $L_{-1} \phi_{1,2}\left(h=\frac{19}{20}\right)$ than to that of the primary operator itself $\left(h=-\frac{1}{20}\right)$. Therefore this result is not surprising. On the other hand this indicates that when studying more complex systems, where the dimensions of the primary operators differ by several units (as it is the case for example for the unitary minimal models), this counting procedure might become rather complex, since different primary operators will mix with descendent operators at various levels.

For general $N$ we use again generating functions $F_{N}$ and $G_{N}$ now defined as

$$
F_{N}^{o} \equiv \sum_{n, o d d} \frac{q^{\frac{n(n+2)}{4}-N n}}{(q)_{n}} \quad \text { and } \quad G_{N}^{e} \equiv \sum_{n, \text { even }} \frac{q^{\frac{n^{2}-1}{4}-N n}}{(q)_{n}}
$$

Observe that they have the same functional form as the generating functions for the even sector (4.10) and (4.11), but the summation over odd and even indices is interchanged. They satisfy the same recursion relations (4.12) but with the initial conditions $F_{0}^{o}=$ $q^{\frac{3}{4}} \tilde{\chi}_{1,4}(q)$ and $G_{0}^{e}=q^{-\frac{1}{4}} \tilde{\chi}_{1,2}$. This implies that the space of operators can be decomposed as

$$
F_{\infty}^{o}=q^{-\frac{1}{4}} \tilde{\chi}_{1,2}(q) \tilde{\chi}_{1,3}(\bar{q})+q^{\frac{3}{4}} \tilde{\chi}_{1,4}(q) \tilde{\chi}_{1,1}(\bar{q})
$$

We have calculated the form factors of the primary operators explicitly, and they are given by the polynomials $Q_{n}$ in (4.4)

$$
Q_{n}=\left\|\sigma_{4 i-2 j-1}\right\|^{<\frac{n-1}{2}>} \quad, \quad n=1,3, \ldots,
$$

for the field $\left(-\frac{1}{20}, \frac{1}{5}\right)$ where the upper index of the determinant indicates the dimension of the corresponding matrix. For the operator $\left(\frac{3}{4}, 0\right)$ the polynomials are

$$
Q_{1}=\sigma_{1} \quad \text { and } \quad Q_{n}=\sigma_{n}\left\|\sigma_{4 i-2 j}\right\|^{<\frac{n-3}{2}>}, n=3,5, \ldots .
$$


We now turn our attention to the sector $w=\frac{1}{2}$. The recursion function (4.7) takes the form

$$
D_{n}=\sum \sigma_{k} x^{n-k} \cos \frac{\pi}{2}(n-k)
$$

The counting argument determines the space of solutions, as being generated by

$$
G_{0}^{e}=\sum_{n, \text { even }} \frac{q^{\frac{n^{2}}{4}}}{(q)_{n}}=\tilde{\chi}_{1,2}(q)
$$

In order to understand the structure of the space of operators we also analyse explicitly the terms with $N=1$. It is given by

$$
G_{1}^{e}=\sum_{n, \text { even }} \frac{q^{\frac{n^{2}}{4}-1}}{(q)_{n}}=(1+\bar{q}) \tilde{\chi}_{1,2}(q)+\tilde{\chi}_{1,4}(q)
$$

that is $\phi_{1,4}$ does not mix with the primary operator $\phi_{1,2}$ but again with its first descendent. We have drawn a picture of the perturbed space of operators in figure 2 .

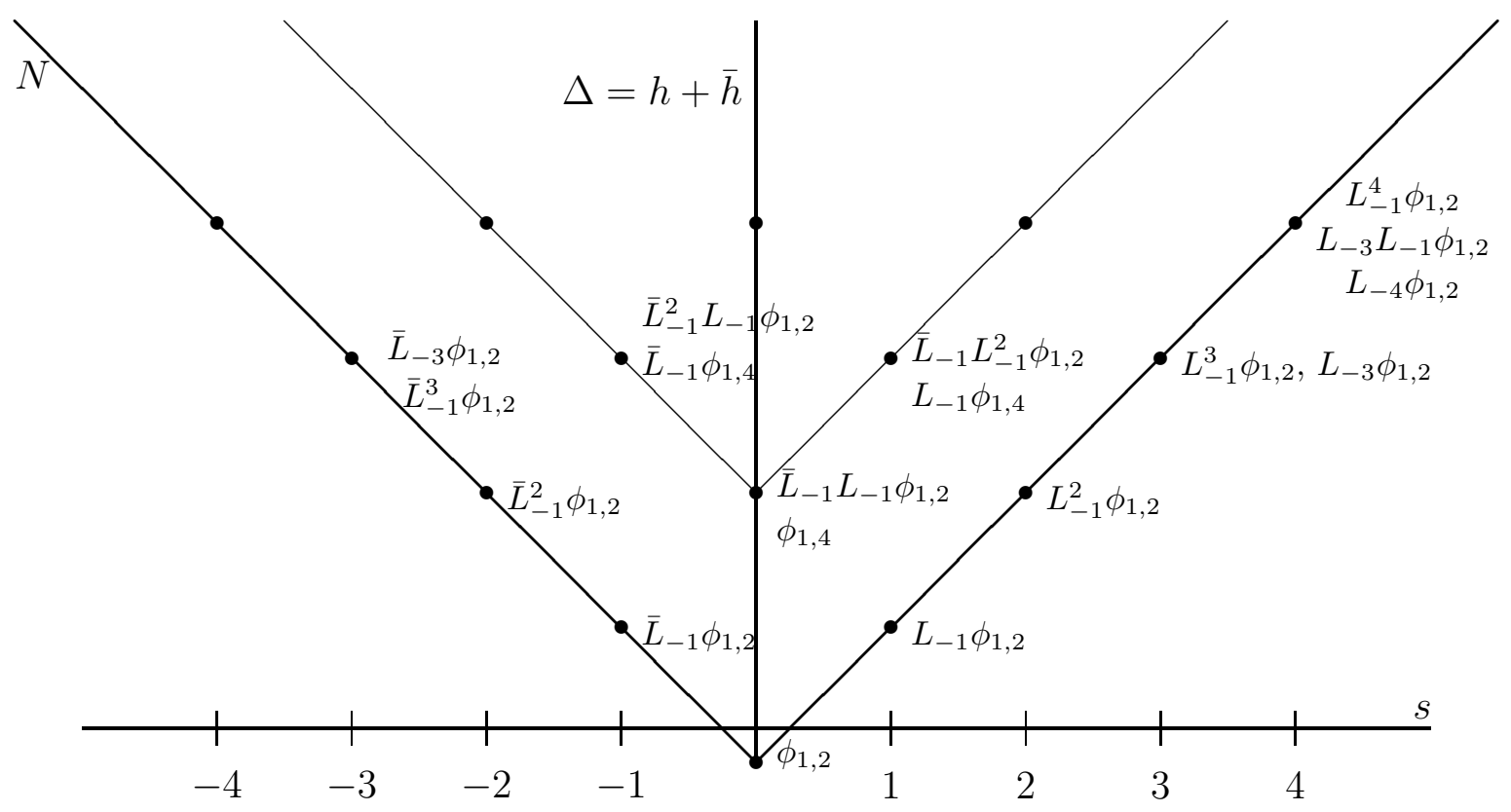

Figure 2: Space of descendent operators in the massive model $\mathcal{M}_{3,5}+\phi_{1,3}$ for the sector $w=\frac{1}{2}$.

The full space of operators is generated by $G_{n}^{e}$ which becomes in the limit $n \rightarrow \infty$

$$
G_{\infty}^{e}=\tilde{\chi}_{1,2}(q) \tilde{\chi}_{1,2}(\bar{q})+q \bar{q} \tilde{\chi}_{1,4}(q) \tilde{\chi}_{1,4}(\bar{q})
$$


The form factors of the primary operator $\left(-\frac{1}{20},-\frac{1}{20}\right)$ can be calculated explicitly, and are given by

$$
Q_{n}=\left\|\sigma_{4 i-2 j}\right\|^{<\frac{n}{2}-1>}, \quad n=2,4, \ldots
$$

in (4.4). In this example it becomes clear that the expressions describing the content of operators in a specific sector in terms of the characters have to be regarded as formal ones. Especially in (4.23) there appears the term $q \bar{q}$ which multiplies the characters of the field $\phi_{1,4}$. If we cancel them, we would not alter (4.23), but the expression would no longer reflect the actual structure of the space of operators in this sector.

Finally let us summerise the analysis of the odd form factors in the sector $w=\frac{1}{2}$. We find that the space is structured as

$$
G_{\infty}^{o}=q^{\frac{1}{4}} \tilde{\chi}_{1,3}(q) \tilde{\chi}_{1,2}(\bar{q})+q^{-\frac{3}{4}} \tilde{\chi}_{1,1}(q) \tilde{\chi}_{1,4}(\bar{q})
$$

The form factors of the primary fields are

$$
Q_{n}=\left\|\sigma_{4 i-2 j-1}\right\|^{<\frac{n-1}{2}>}, \quad n=1,3, \ldots
$$

for the operator $\left(\frac{1}{5},-\frac{1}{20}\right)$ and

$$
Q_{1}=\bar{\sigma}_{1} \quad \text { and } \quad Q_{n}=\left\|\sigma_{4 i-2 j+1}\right\|^{<\frac{n-3}{2}>}, n=3,5, \ldots
$$

for the operator $\left(0, \frac{3}{4}\right)$.

Let us discuss the physical implications of the above analysis. The massive model $\mathcal{M}_{3,5}+\phi_{1,3}$ contains the following primary operators: the scalar fields $\left(\frac{1}{5}, \frac{1}{5}\right)$ and $(0,0)$ and the para-fermionic fields $\left(s=-\frac{1}{4}+\mathbf{Z}\right)$, namely $\left(-\frac{1}{20}, \frac{1}{5}\right),\left(\frac{3}{4}, 0\right)$ in the sector $w=0$. In the sector $w=\frac{1}{2}$ we find the fields $\left(-\frac{1}{20},-\frac{1}{20}\right),\left(\frac{3}{4}, \frac{3}{4}\right)$ and the spin $s=\frac{1}{4}+\mathbf{Z}$ operators $\left(\frac{1}{5},-\frac{1}{20}\right),\left(0, \frac{3}{4}\right)$. We want to stress the fact, that we are not able to establish a direct link between the form factors of these massive operators and their conformal counterparts, but have rather defined those operators through the degeneracies of their descendent spaces.

An interesting feature of this model is the appearance of the para-fermionic operators. Their spin is determined by the spin of the asymptotic states. This causes that only specific combinations of chiral dimensions can appear in the massive model. Thus in 
contrast to the space of operators in the conformal theory where arbitrary combinations of right and left dimensions can occur for a theory defined on the real line.

We have introduced the chiralities in the massive model through the variables $x$ and $\bar{x}=\frac{1}{x}$. Therefore, in order to have a consistent description we should be able to transform the form factors of the operator $\left(-\frac{1}{20}, \frac{1}{5}\right)$ to those of the operator $\left(\frac{1}{5},-\frac{1}{20}\right)$ by the transformation $x \rightarrow \bar{x}$ (and similarly for the other pair of para-fermionic fields). Let us analyse the form factors of $\left(-\frac{1}{20}, \frac{1}{5}\right)$, given by

$$
\mathcal{F}_{n}^{\left(-\frac{1}{20}, \frac{1}{5}\right)}=H_{n} \sigma_{n}^{\frac{n}{2}-\frac{1}{2}}\left\|\sigma_{4 i-2 j}\right\|^{<\frac{n-1}{2}>} \prod \frac{F^{\min }\left(\beta_{i j}\right)}{\left(x_{i}+x_{j}\right)} .
$$

We define $\overline{\mathcal{F}}_{n}\left(\beta_{1}, \ldots, \beta_{n}\right)=\mathcal{F}_{n}\left(-\beta_{1}, \ldots,-\beta_{n}\right)$. Using the relation (1.14) we find for the determinant

$$
\left\|\bar{\sigma}_{4 i-2 j}\right\|^{<\frac{n-1}{2}>}=\sigma_{n}^{-\frac{n-1}{2}}\left\|\sigma_{4 i-2 j-1}\right\|^{<\frac{n-1}{2}>} \quad,
$$

and therefore

$$
\overline{\mathcal{F}}_{n}^{\left(-\frac{1}{20}, \frac{1}{5}\right)}=\mathcal{F}_{n}^{\left(\frac{1}{5},-\frac{1}{20}\right)}
$$

Analogously one can show that $\overline{\mathcal{F}}_{n}^{\left(0, \frac{3}{4}\right)}=\mathcal{F}_{n}^{\left(\frac{3}{4}, 0\right)}$.

Related to this fact is the following observation. In calculating the form factors, we had to choose which chirality i.e. which limit of the $S$-matrix to select in the analytic continuation process. We chose the limit $\beta \rightarrow \infty$. Suppose we would choose $S(-\infty)=+i$. Repeating the same analysis as before, one finds that the scalar fields remain in the respective sectors, while the spin-dependent fields change place. This is as expected, since now the chirality of the spin is now linked to the right component of the field dimensions.

A final remark concerns the scalar operators of the theory. We have found $\phi_{1,1}$ and $\phi_{1,3}$ in the sector $w=0$ while we found $\phi_{1,2}$ and $\phi_{1,4}$ in the sector $w=\frac{1}{2}$. It is interesting to note that these locality properties are in accordance with the operator-product expansions of the correspondent conformal operators with the field $\phi_{1,4}$. It has been observed previously [27] that the field $\phi_{2,1}$ (which for $\mathcal{M}_{3,5}$ is the same as $\phi_{1,4}$ ) is deeply connected to the operators generating the asymptotic states. This fact is not totally understood. The analysis of more complex theories in the formalism proposed in this article might give new insights to this problem. 


\section{The models $M_{2,2 p+3}$}

From our point of view, the models $\mathcal{M}_{2,2 p+3}$ constitute the simplest systems containing more than one asymptotic particle, since their Kac table contains only one row of fields. Also their spectrum contains only scalar particles, and from the analysis of the previous sections we expect only scalar operators to appear in the massive model, as it was also the case for the Yang-Lee model.

The $S$-matrices are given by 23

$$
S_{a b}=f_{|a-b| \frac{\alpha}{2}}(\beta) f_{(a+b) \frac{\alpha}{2}}(\beta) \prod_{k=1}^{\min (a, b)-1}\left(f_{(|a-b|+2 k) \frac{\alpha}{2}}(\beta)\right)^{2}
$$

where $\alpha=\frac{2 \pi}{2 p+1}$, and $a, b=1,2, \ldots p$ label the particles of mass $m_{a}=\sin \left(a \frac{\alpha}{2}\right)$. The functions $f$ are given by

$$
f_{\alpha}(\beta) \equiv \frac{\tanh \frac{1}{2}(\beta+i \alpha)}{\tanh \frac{1}{2}(\beta-i \alpha)}
$$

Note that the $S$-matrix of the fundamental particle has the same functional structure as the $S$-matrix of the Yang-Lee model, the difference being that the parameter $\alpha$ is specified differently. We can therefore use the results from section 3, where we had analysed the kinematical recursion relation for the form-factors corresponding to this $S$-matrix element for general $\alpha$.

The form factor equations for the models $\mathcal{M}_{2,2 p+3}+\phi_{1,3}$ have been analysed in detail in [22]. There the form factors for all perturbed primary operators $\phi_{1, k}, k=1 \ldots, p$ have been calculated explicitly. They are given by (3.3) with $Q_{n}=\mathcal{Q}_{n}(k)$ where $\mathcal{Q}_{n}(k)$ is defined in (3.9). Further it was shown that there is only one independent recursion relation resulting from all possible bound state fusion processes. This recursion relation can be expressed as a constraint on the form factors with only indices $1 \ldots 1$ relating $F_{n+2 p+1}$ to $F_{n}$. It follows that the consistent form factors of this model form a subset of the solutions of the kinematical recursion relation on particle 1. This allows us to determine the structure of the form factors of higher particles by carrying out the bootstrap on the expression obtained for $F_{1,1, \ldots, 1}$.

We carry out the bootstrap explicitly for the form factors involving particles one and 
two. They are given by

$$
\begin{gathered}
\mathcal{F}_{2,2, \ldots, 2} \underbrace{1,1, \ldots, 1}_{r}\left(\beta_{1}, \ldots, \beta_{q+r}\right)= \\
H_{2 q+r} Q_{2,2 \ldots 2,1,1 \ldots 1} \prod_{m=1}^{q} A\left(x_{m}\right) \prod_{i<j}^{r} B_{1,1}\left(\beta_{i j}\right) \prod_{m=1}^{q} \prod_{i=1}^{r} B_{1,2}\left(\beta_{m i}\right) \prod_{m<l}^{q} B_{2,2}\left(\beta_{m l}\right)
\end{gathered}
$$

The single components in this complex expression are

$$
\begin{gathered}
B_{1,1}(\beta)=\frac{(-i) \sinh \left(\frac{\beta}{2}\right) \zeta_{11}(\beta)}{\left(x_{i}+x_{j}\right)<\alpha>}, \\
B_{1,2}(\beta)=\frac{-\zeta_{12}(\beta)}{4 x_{m} x_{i} \zeta(i \pi, \alpha)^{2}<1-\frac{\alpha}{2}><\frac{3}{2} \alpha>}, \\
B_{2,2}(\beta)=\frac{(-i) \sinh \frac{\theta}{2} \zeta_{22}(\beta)}{4 x_{m} x_{n} \pi \zeta(i \pi, \alpha)^{4}<2 \alpha><\alpha><1-\alpha>\left(x_{m}+x_{n}\right)^{2}}
\end{gathered} .
$$

$H_{n}$ is the normalisation constant of $F_{1,1, \ldots, 1}$ and

$$
A(x)=-\cot \alpha \tan \frac{\alpha}{2} \frac{1}{\sqrt{\pi \zeta(i \pi, 2 \alpha)} \zeta(i \pi, \alpha) 2 \pi} \quad .
$$

Further we have introduced an abbreviated expression for the functions introducing the bound state poles,

$$
<\alpha>\equiv \sinh \frac{1}{2}\left(\beta_{i j}-i \alpha\right) \sinh \frac{1}{2}\left(\beta_{i j}+i \alpha\right)
$$

and the minimal 2 particle form factors

$$
\begin{aligned}
& \zeta_{11}(\beta)=\zeta(\theta, \alpha), \\
& \zeta_{12}(\beta)=\zeta\left(\theta, \frac{\alpha}{2}\right) \zeta\left(\theta, \frac{3 \alpha}{2}\right), \\
& \zeta_{22}(\beta)=\zeta(\theta, 2 \alpha) \zeta(\theta, \alpha)^{2},
\end{aligned}
$$

with

$\zeta(\beta, \alpha)=\prod_{k=0}^{\infty} \frac{\Gamma\left(k+\frac{1}{2}+\frac{\alpha}{2 \pi}-\frac{i \beta}{2}\right) \Gamma\left(k+1-\frac{\alpha}{2 \pi}-\frac{i \beta}{2}\right)}{\Gamma\left(k+\frac{1}{2}-\frac{\alpha}{2 \pi}-\frac{i \beta}{2}\right) \Gamma\left(k+\frac{\alpha}{2 \pi}-\frac{i \beta}{2}\right)} \frac{\left.\Gamma+\frac{3}{2}+\frac{\alpha}{2 \pi}+\frac{i \beta}{2}\right) \Gamma\left(k+2-\frac{\alpha}{2 \pi}+\frac{i \beta}{2}\right)}{\Gamma\left(k+\frac{3}{2}-\frac{\alpha}{2 \pi}+\frac{i \beta}{2}\right) \Gamma\left(k+1+\frac{\alpha}{2 \pi}+\frac{i \beta}{2}\right)}$.

We start our investigation by analysing the system $\mathcal{M}_{2,7}$. The above expressions are sufficient to examine the operator content of this model, since it contains only two particles. Let us analyse the form factors corresponding to chiral operators, i.e. $N=0$ in (1.13). This implies that the functions $Q_{2,2 \ldots 2,1,1 \ldots 1}$ are polynomials. 


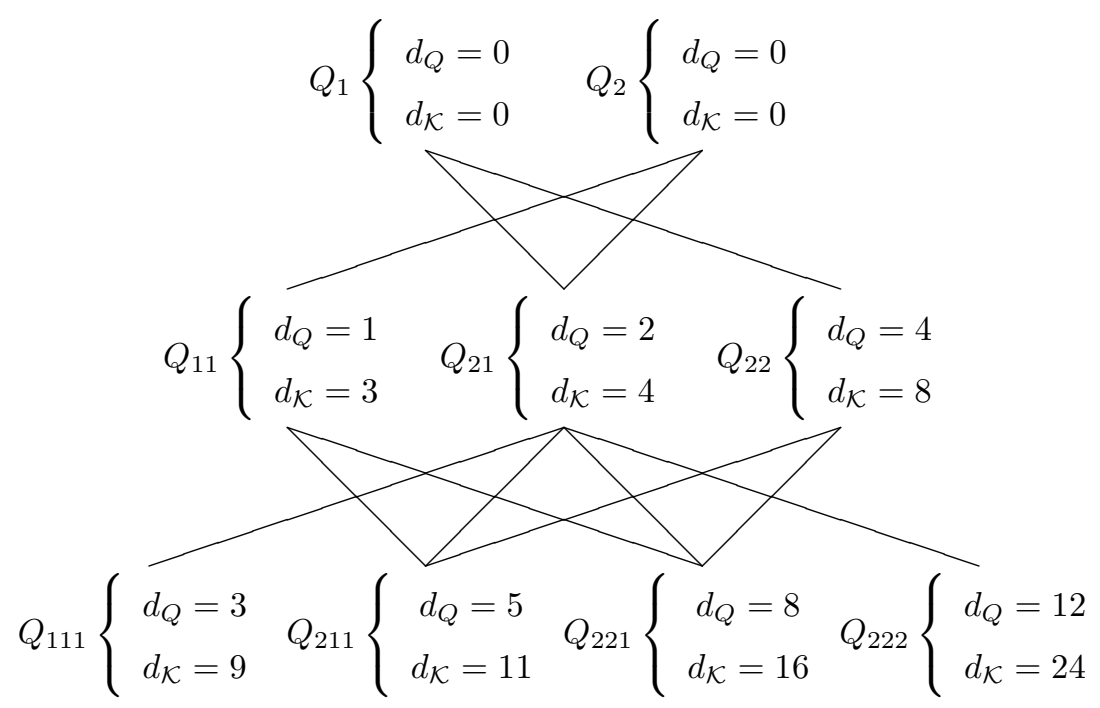

Figure 3: Comparing dimensions of $Q_{\epsilon_{1}, \ldots, \epsilon_{n}}$ with the respective kernels of the recursion relations. The lines indicate the inter-relations due to the bound state equation (1.9).

As before, we apply the counting technique, but now we have to take in account that we treat a two-particle system. The recursion problem for the functions $Q_{\epsilon_{1}, \ldots \epsilon_{n}}$ is now of matrix-form. We need to determine the respective degrees of the polynomials $Q_{\epsilon_{1}, \ldots \epsilon_{n}}$ and of the kernel of the recursion relation. The degrees of $Q_{2,2 \ldots 2,1,1 \ldots 1}$ follow from Lorentz invariance, as

$$
\operatorname{deg}(Q_{q}^{2,2, \ldots, 2} \underbrace{1,1, \ldots, 1}_{r})=\frac{1}{2}(2 q+r)(2 q+r-1)-q+s \quad,
$$

where $s$ denotes the spin of the operator as usual. The degrees of the kernels of the recursion relations, $\mathcal{K}_{2,2, \ldots, 2,1,1, \ldots, 1}$ follow from the pole structure of $(5.4)-(5.6)$, and are given by

$$
\operatorname{deg}\left(\mathcal{K}_{2,2, \ldots, 2}^{2,1, \ldots, 1}\right)=\frac{3}{2} r(r-1)+4 r q+4 q(q-1)
$$

We have depicted these dimensions in figure 3. As in the one particle systems we use this comparison of degrees in order to count the number of independent solutions of the recursion relations. Note that the polynomials $Q_{\epsilon_{1}, \ldots \epsilon_{n}}$ are symmetric in coinciding indices only. Therefore in this example we have two sets of indices, which have to be taken in account separately. From figure 3 we immediately find that the first solutions 
are being generated by

$$
\frac{1}{(q)_{1}}+\frac{1}{(q)_{1}}
$$

which correspond to the possible choices of one-particle form factors. The terms

$$
\frac{q^{2}}{(q)_{2}}+\frac{q^{2}}{(q)_{1}(q)_{1}}+\frac{q^{4}}{(q)_{2}}
$$

generate the number of independent solutions for the two-particle form factors. The power of $q$ in the nominator derives from the spin-value at which $\operatorname{deg}\left(Q_{\epsilon_{1}, \epsilon_{2}}\right)+s=\operatorname{deg}\left(\mathcal{K}_{\epsilon_{1}, \epsilon_{2}}\right)$. In particular, the denominator $(q)_{1}(q)_{1}$ corresponds to $Q_{21}\left(x_{1}, x_{2}\right)$ and indicates that this function is not symmetric with respect to its two arguments. Finally for the three-particle form factors we find

$$
\frac{q^{6}}{(q)_{3}}+\frac{q^{6}}{(q)_{2}(q)_{1}}+\frac{q^{8}}{(q)_{1}(q)_{2}}+\frac{q^{12}}{(q)_{3}} .
$$

In order to extract the general generating function, one uses the fact, that the spin value at which a new solution enters is given by $\operatorname{deg}\left(\mathcal{K}_{\epsilon_{1}, \ldots \epsilon_{n}}\right)-\operatorname{deg}\left(Q_{\epsilon_{1}, \ldots \epsilon_{n}}\right)$. Defining

$$
F_{a, b} \equiv \sum_{n_{1}, n_{2}} \frac{q^{\left(n_{1}+n_{2}\right)^{2}+n_{2}^{2}-a\left(n_{1}+n_{2}\right)-b n_{2}}}{(q)_{n_{1}}(q)_{n_{2}}}
$$

and using (5.10) and (5.11) one finds that the space of chiral operators in the $\phi_{1,3}$ perturbation of $\mathcal{M}_{2,7}$ is generated by $F_{1,1}$. The functions (5.15) satisfy

$$
\begin{aligned}
& F_{a, b}=F_{a, b-1}+\bar{q}^{a+b-2} F_{a-2, b-2}, \\
& F_{a, b}=F_{a-1, b+1}+\bar{q}^{a-1} F_{a-2, b} .
\end{aligned}
$$

These recursion relations can be used to prove that

$$
F_{1,1}=F_{-1,-1}+F_{0,-1}+F_{0,0}=\tilde{\chi}_{1,1}(q)+\tilde{\chi}_{1,2}(q)+\tilde{\chi}_{1,3}(q)
$$

As in the previous cases we find that the chiral operator content of the massive model is in a one-to-one correspondence with that of the conformal model.

Unfortunately the results for this model are less complete than the others. We have tried to solve the recursion relations (5.16) to find an exact expression for the whole space of operators (given by $\lim _{N \rightarrow \infty} F_{N, N}$ ) of the model, but did not succeed. Nevertheless we have solved them numerically up to order 50, and the results indicate that

$$
F_{N, N}=\tilde{\chi}_{1,1}(q) \tilde{\chi}_{1,1}(\bar{q})+\tilde{\chi}_{1,2}(q) \tilde{\chi}_{1,2}(\bar{q})+\tilde{\chi}_{1,3}(q) \tilde{\chi}_{1,3}(\bar{q})\left(\bmod q^{N}\right)
$$


as expected.

Finally let us turn to the general systems $\mathcal{M}_{2,2 p+3}$. Again we need to determine the dimensions of $Q_{\epsilon_{1}, \ldots, \epsilon_{n}}$ and the kernel $\mathcal{K}_{\epsilon_{1}, \ldots, \epsilon_{n}}$. Denote as $Q^{m_{1}, m_{2}, \ldots, m_{p}}$ the function $Q_{\epsilon_{1}, \ldots, \epsilon_{n}}$ corresponding to $m_{i}$ indices $\epsilon=i$, where $i=1, \ldots, p$, the number of different asymptotic states in the theory. From Lorentz invariance one finds that

$$
\operatorname{deg}\left(Q^{m_{1}, m_{2}, \ldots, m_{p}}\right)=\frac{1}{2}\left(\sum_{i=1}^{p} i m_{i}\right)\left(1+\sum_{i=1}^{p} i m_{i}\right)-\sum_{i=1}^{p} \frac{1}{2} i(i-1) m_{i}
$$

For example the dimension of $Q_{k l}\left(x_{1}, x_{2}\right)$, i.e. the function corresponding to the twoparticle form factor of particles $k$ and $l$, has dimension $\operatorname{deg}\left(Q_{k l}\right)=k l$.

In order to determine the dimension of the kernel we must carry out the bootstrap on the form factors of particle 1, satisfying the kinematical recursion relation. This is straightforward for the denominators and for the factor $\sinh \left(\frac{\beta}{2}\right)$ in (5.4). But one needs to be careful, since further zeros appear from the bootstrap of the factor $\zeta_{11}$. For example for the two particle form factor $\mathcal{F}_{k l}$ with $k>l$ one finds through the bootstrap that

$$
\begin{aligned}
\zeta_{1,1} \rightarrow\left(\sinh \frac{\beta}{2}\right)^{\delta_{n, k}} & \zeta\left(\beta,(k-l) \frac{\alpha}{2}\right) \zeta\left(\beta,(k+l) \frac{\alpha}{2}\right) \prod_{i=1}^{l-1} \zeta\left(\beta,(k-l+2 i) \frac{\alpha}{2}\right) \times \\
& \prod_{i=2}^{k-1} \prod_{j=0}^{l-1} \zeta\left(\beta,(k+l) \frac{\alpha}{2}-(i+j) \alpha\right)
\end{aligned}
$$

The first line in (5.19) gives exactly the minimal two-particle form factor for the particles $k$ and $l, \zeta_{k l}$, while the second line reduces to trigonometric functions, due to the relation $\zeta(\beta, \alpha) \zeta(\beta,-\alpha)=<\alpha>$. They cancel poles which arise from the bootstrap of the poles in the denominator of (5.4).

We will not give a detailed account of this calculation. Carrying out the bootstrap on all factors in (3.3) one finds that the total degree of the kernel of the recursion relations corresponding to the function $Q_{k l}$ is given by $\operatorname{deg}\left(\mathcal{K}_{k l}\right)=k . l+2 . l$. Comparing with the dimensions of $Q_{k l}$, one finds that solutions corresponding to the kernel of $\mathcal{K}_{k l}$ will enter at level $2 l$ (recall we have the convention that $k>l$ ). Therefore the general exponent in the $q$-sum expression will be given by

$$
\begin{aligned}
& 2 l n_{k} n_{l} \quad \text { for } k>l, \\
& k n_{k}\left(n_{k}-1\right) \quad \text { for } k=l,
\end{aligned}
$$


for every pair of indices $n_{k}$ and $n_{l}$. Taking the sum over all particles we find

$$
\sum_{k=1}^{p}\left(k n_{k}\left(n_{k}-1\right)+\sum_{l<k} 2 \ln _{l} n_{k}\right)=\sum_{k=1}^{p} N_{k}^{2}-N_{k}
$$

where we defined $N_{j}=\sum_{i=0}^{p-j} n_{p-i}$. This leads to the final expression for chiral massive states as

$$
F_{1,1, \ldots, 1}=\sum_{n_{1}, \ldots n_{p}} \frac{q^{N_{1}^{2}-N_{1}+N_{2}^{2}-N_{2}+\ldots+N_{p}^{2}-N_{p}}}{(q)_{n_{1}}(q)_{n_{2}} \ldots(q)_{n_{p}}} .
$$

We want to show that this sum expression coincides with a sum over characters of the model. For that we need the $q$-sum expressions of the characters, given through the Gordon-Andrews identities (see e.g. [12]) as

$$
\tilde{\chi}_{1, j}(q)=F_{j-1}^{00 \ldots 0} \underbrace{-1-1 \ldots-1}_{p+1-j}
$$

where we have introduced the generating functions

$$
F_{k_{1}, \ldots, k_{p}} \equiv \sum_{n_{1}, \ldots n_{p}} \frac{q^{N_{1}^{2}-k_{1} N_{1}+N_{2}^{2}-k_{2} N_{2}+\ldots+N_{p}^{2}-k_{p} N_{p}}}{(q)_{n_{1}}(q)_{n_{2}} \ldots(q)_{n_{p}}},
$$

which satisfy the recursion identities

$$
\begin{gathered}
F_{k_{1}, \ldots, k_{p}}=F_{k_{1}, \ldots, k_{i-1}, k_{i}-1, k_{i+1}+1, k_{i+2}, \ldots, k_{p}}+ \\
\bar{q}^{k_{1}-1} \bar{q}^{k_{2}-1} \ldots \bar{q}^{k_{i}-1} F_{k_{1}-2, k_{2}-2, \ldots, k_{i}-2, k_{i+1}, \ldots, k_{p}}
\end{gathered}
$$

Apply these identities to the generating function of the chiral operators, namely

$$
F_{11 \ldots 1}=F_{111 \ldots 10}+F_{-1-1 \ldots-1-1} \quad .
$$

The last factor corresponds to $\tilde{\chi}_{1,1}(q)$. Now we have to continue with

$$
F_{11 \ldots 110}=F_{11 \ldots 01}+F_{-1-1, \ldots-10}
$$

This last term does not correspond to any conformal character, but if we use the recursion relations to permute through the 0 to stand at the first position we find $\tilde{\chi}_{1,2}(q)$ along with a plethora of terms, without physical interpretation. If one carries out this commutation process of generating always further zeros as the last index and commuting them through, one will finally end up with

$$
F_{11 \ldots 1}=\sum_{i=1}^{p} \tilde{\chi}_{1, i}^{(2,2 p+3)}(q)
$$


We conclude that in this series of models the operator content of the massive theory coincides with the content of conformal scalar operators. Unfortunately in all these systems, containing more than one asymptotic state, we were not able to solve the general recursion-relations (5.24) which determine the full space of operators of the massive model. We have done several numerical calculations which lead us to the conjecture that the full space of operators in the massive model corresponds to the scalar conformal one, i.e.

$$
Z=\sum_{i=1}^{p} \tilde{\chi}_{1, i}(q) \tilde{\chi}_{1, i}(\bar{q})
$$

We have also compared the structure of the solutions of these recursion relation with several known 'finitized' expressions for the characters of these models [29], but were not able to find one which provides a solution to the recursion problem (5.24).

\section{Conclusions}

The aim of this work was to analyse the structure of the space of operators in massive integrable systems. Our particular interest was in deformed minimal conformal field theories, because these theories at the critical point have a well-known structure, that is the operators are organised into families determined through the Virasoro irreducible representations, with a highest weight state corresponding to a primary operator with rational conformal weight. All other operators can be obtained by applying the generators of the Virasoro algebra to these primary fields.

For massive integrable models such an algebraic analysis has not yet been developed. As an alternative we have used the form factor bootstrap method in order to determine the structure of the space of operators. In order to find similarities we have used the physical properties of the conformal operators and have searched for analogues in the massive models. In particular we have introduced a grading into our space of operators in the massive model defining chiral operators as those with the mildest ultraviolet behaviour and through their spin. The whole space of operators is constructed by augmenting the divergence of the form factors gradually.

Using the recursive equations for the form factors we have proposed a counting proce- 
dure in order to find the number of operators in each of the finite dimensional subspaces defined through this grading. Though we have analysed only simple systems (in the sense that the Kac-table of the corresponding conformal model consisted at the most of two rows), the method is applicable to any system described through a factorised scattering theory, including systems with degenerate particles and even massless scattering theories, which describe the flow from one critical point to another.

Our analysis of the massive models gives several interesting results. For all the systems examined the chiral field content is in a one-to-one correspondence with that of the underlying conformal theory. The massive primary operators are grouped into families according to the parity of the corresponding conformal field. This induces the possibility of a mixing of the descendent operators in the corresponding Virasoro irreducible representations in the process of perturbation. The full space of space of operators in the massive model contains two types of fields. For theories with scalar asymptotic states there are only scalar fields, while for models with asymptotic particles which satisfy non-trivial statistics, in addition to the scalar fields also para-fermionic operators are present in the theory. This generates for example in the model $\mathcal{M}_{3,5}$ operators satisfying para-statistics with fractional spin $\frac{1}{4}$.

Finally let us comment on the mathematical methods involved. A feature, which persisted in all models examined, was that the counting of the operators lead naturally to fermionic sum expressions of the characters, or rather sums of them. Recently a lot of interest has evolved in this subject, after Kedem et.al. [28] have conjectured a whole series of such character identities, which have been proved in [29, 30, 31], by using finitizations of the character expressions. As we have seen, the counting procedure leads to finite expressions for the characters, when we generate the full space of states. It seems to be an interesting fact, that the method here proposed gives a natural physical application to these mathematical results. 


\section{Acknowledgments}

I am indebted to A. Berkovich, L. Chau, A. Fring, R. Kedem, A. Kent, B. McCoy, G.

Watts and A. Zamolodchikov for discussions, and to A. Recknagel for obtaining some important literature for me. This work was supported by PPARC grant GR/J20661.

\section{References}

[1] A.B. Zamolodchikov, in Advanced Studies in Pure Mathematics 19 (1989), 641; Int. J. Mod. Phys.A3 (1988), 743;

[2] B. Berg, M. Karowski and P. Weisz, Phys. Rev. D19 (1979), 2477; M. Karowski and P. Weisz, Nucl. Phys. B139 (1978), 445; M. Karowski, Phys. Rep. 49 (1979), 229;

[3] F.A. Smirnov, "Form Factors in Completely Integrable Models of Quantum Field Theory", (World Scientific 1992); F. A. Smirnov, in Introduction to Quantum Group and Integrable Massive Models of Quantum Field Theory, Nankai Lectures on Mathematical Physics, World Scientific 1990;

[4] V.P. Yurov and Al. B. Zamolodchikov, Int. J. Mod. Phys. A6 (1991), 3419;

[5] D. Bernard and A. LeClair, Nucl. Phys. B399 (1993), 709;

[6] F.A. Smirnov, J. Phys. A17 (1984), L873; F.A. Smirnov, J. Phys. A19 (1984), L575; A.N. Kirillov and F.A. Smirnov, Phys. Lett. B198 (1987), 506; A.N. Kirillov and F.A. Smirnov, Int. J. Mod. Phys. A3 (1988), 731; Int. J. Mod. Phys. A4 (1989),4231;

[7] J.L. Cardy and G. Mussardo, Nucl. Phys. B340 (1990), 387;

[8] I.G. MacDonald, "Symmetric Functions and Hall Polynomials" (Clarendon Press, Oxford, 1979);

[9] G. Mussardo, Lecture Notes of "Summer School in High Energy Physics and Cosmology" Trieste 1993

[10] P. Christe, Int. J. Mod. Phys. A6 (1991), 5271;

[11] B.L. Feigin and D.B. Fuchs, Funct. Anal. Appl. 17 (1983), 241; A. Rocha-Caridi, in "Vertex Operators in Mathematics and Physics" eds. J. Lepowsky, S. Mandelstam and I.M. Singer (Springer 1985), 451;

[12] G.E. Andrews "The Theory of Partitions" (Addison-Wesley 1976);

[13] H. Rademacher "Topics in Analytic Number Theory" (Springer 1973);

[14] G.E. Andrews, R.J. Baxter and P.J. Forrester, J. Stat. Phys. 35 (1984), 193; R.J. Baxter and P.J. Forrester, J. Stat. Phys. 38 (1985), 435;

[15] P. Bouwknegt, A.W.W. Ludwig and K. Schoutens, Spinon Bases, Yangian symmetry and fermionic representations of Virasoro characters in conformal field theory, USC-94/9, PUPT-1469, hepth/9406020;

[16] R.J. Baxter, "Exactly solved models in statistical mechanics" (Academic Press 1982); J.L. Cardy, in "Fields, strings and critical phenomena" Proceedings Les Houches 1988, eds. E. Brezin and J. Zinn-Justin;

[17] A. Koubek, A method to determine the operator content of perturbed conformal field theories DAMTP-94/89; 
[18] J.L. Cardy and G. Mussardo, Phys. Lett. B225 (1989), 275;

[19] F.A. Smirnov, Nucl. Phys. B337 (1989), 156;

[20] Al.B. Zamolodchikov, Nucl. Phys. B348 (1991), 619;

[21] A. Koubek and G. Mussardo, Phys. Lett. B311 (1993),193;

[22] A. Koubek, Form factor bootstrap and the operator content of perturbed minimal models, DAMTP94/15; hep-th/9405014, to appear in Nucl. Phys. B;

[23] P.G. Freund, T.R. Klassen and E. Melzer, Phys. Lett. B229 (1989), 507;

[24] M. Yu. Lashkevich, Sectors of mutually local fields in integrable models of quantum field theory LANDAU-94-TMP-4; hep-th/9406118;

[25] G. Delfino and G. Mussardo, Phys. Lett. B324 (1994), 40;

[26] N. Reshitikhin and F.A. Smirnov, Commun. Math. Phys. 131 (1990) 157;

[27] F.A. Smirnov, Commun. Math. Phys. 132 (1990), 415;

[28] R. Kedem, T.R. Klassen, B.M. McCoy and E. Melzer, Phys. Lett. B304 (1993), 263; Phys. Lett. B307 (1993), 68;

[29] A.N. Kirillov, Dilogarithm identities, hep-th/9408113; Y.-H. Quano, Polynomial identities of the Rogers-Ramanujan type, Univ. of Melbourne preprint No. 25, hep-th/9407191: O. Foda and Y.-H. Quano, Virasoro character identities from the Andrews-Bailey construction, hep-th/9408086;

[30] E. Melzer, Int. J. Mod. Phys. A9 (1994), 1115;

[31] A. Berkovich, Fermionic counting of RSOS-states and Virasoro character formulas for the unitary minimal series $\mathcal{M}_{\nu, \nu+1}$. Exact results, Bonn-HE-94-04, hep-th/9403073; 